\title{
THE GALACTIC CENTER ENVIRONMENT
}

\author{
Mark Morris \\ University of California at Los Angeles, Los Angeles, California 90095-1562 \\ Eugene Serabyn \\ California Institute of Technology, Pasadena, California 91125
}

KEY WORDS: Galactic bar, magnetic fields, SgrA*, circumnuclear disk, star formation, molecular clouds, black holes, $\mathrm{X}$ rays

\begin{abstract}
The central half kiloparsec region of our Galaxy harbors a variety of phenomena unique to the central environment. This review discusses the observed structure and activity of the interstellar medium in this region in terms of its inevitable inflow toward the center of the Galactic gravitational potential well. A number of dissipative processes lead to a strong concentration of gas into a "Central Molecular Zone" of about 200-pc radius, in which the molecular medium is characterized by large densities, large velocity dispersions, high temperatures, and apparently strong magnetic fields. The physical state of the gas and the resultant star formation processes occurring in this environment are therefore quite unlike those occurring in the large-scale disk. Gas not consumed by star formation either enters a hot X ray-emitting halo and is lost as a thermally driven galactic wind or continues moving inward, probably discontinuously, through the domain of the few parsec-sized circumnuclear disks and eventually into the central parsec. There, the central radio source SgrA* currently accepts only a tiny fraction of the inflowing material, likely as a result of a limit cycle wherein the continual inflow of matter provokes star formation, which in turn can temporarily halt the inflow via mass-outflow winds.
\end{abstract}

\section{INTRODUCTION}

In its most inclusive sense, the Galactic center encompasses a wide variety of phenomena occurring on stellar to galactic scales. Because of this, and because 
research in this area has advanced rapidly for almost three decades, volumes could be written to summarize our current state of knowledge of this region. Indeed, four conference volumes dedicated largely to this subject can now be consulted (Riegler \& Blandford 1982, Backer 1987, Morris 1989, Genzel \& Harris 1994), and the pages of this review have already seen three major summaries on Galactic center research: those of Oort (1977), Brown \& Liszt (1984), and Genzel \& Townes (1987). In related contributions, Frogel (1988) discusses the stellar content of the Galactic bulge and Reid (1993) summarizes research on the distance to the Galactic center; here, we adopt his suggested distance of $8.0 \pm 0.5 \mathrm{kpc}$. The most recent reviews on the Galactic center are those by Blitz et al (1993) and Genzel et al (1994).

Given this plethora of both information and publications, we have chosen to concentrate this review on the interstellar environment and, in particular, on the causes and ramifications of radial mass flow toward the center. This encompasses the nature of the central molecular layer, the hot medium surrounding it, and the strong magnetic field in which they are found (Section 2); the central stellar bar and its effect on the gas (Section 3); star formation (Section 4); the nature and fate of the circumnuclear gas disk (Section 5); and the central nuclear radio source, Sagittarius A* (Section 6). In the final section we address the possibility of recurrent, highly energetic activity in the Galactic nucleus. Timely topics notably missing from this review include the compact sources of high-energy radiation near the Galactic center, large-scale jets, stellar populations and distributions within the Galactic bulge, stellar kinematics, collisions and mass segregation in the central stellar core, and determinations of the radial mass distribution beyond the central few parsecs. Recent information on these subjects can be found in previous reviews and in Sofue et al (1989), Sofue (1994), Lee (1994, 1995), and Blitz \& Teuben (1996).

\section{CONTENTS OF THE INTERSTELLAR MEDIUM}

\subsection{The Arena}

The emissivity of our Galaxy's core reflects a unique environment. Line emission from both molecular CO and atomic C peak sharply in our Galaxy's central few hundred parsecs, and a somewhat smaller region is quite bright in radio and infrared continuum emission as well (Figure 1; Altenhoff et al 1978, Mezger \& Pauls 1979, Odenwald \& Fazio 1984, Dame et al 1987, Handa et al 1987, Cox \& Laureijs 1989, Bennett et al 1994). This compact and luminous nuclear region, hereafter designated the central molecular zone or CMZ (to distinguish this largely molecular region from the more extensive "H I nuclear disk" in which it is ensconced), produces $\sim 5-10 \%$ of our Galaxy's infrared and 
Lyman continuum luminosities and accounts for roughly $10 \%$ of our Galaxy's molecular gas content (Mezger 1978, Mezger \& Pauls 1979, Hauser et al 1984, Scoville \& Sanders 1987, Cox \& Laureijs 1989, Güsten 1989, Scoville \& Good 1989, Bloemen et al 1990, Wright et al 1991, Bennett et al 1994). The Galactic center is obscured at optical and UV wavelengths by a line-of-sight interstellar extinction of roughly 30 visual magnitudes, but becomes accessible again at energies above $\sim 1 \mathrm{keV}$, where $\mathrm{X}$-ray line emission again peaks strongly on the Galactic center (Koyama et al 1989, Yamauchi \& Koyama 1993). In contrast, the CMZ does not stand out in the continuum at high energies (Blitz et al 1985, Bloemen et al 1986, Cook et al 1991, Yamauchi \& Koyama 1993, Diehl 1994, Goldwurm et al 1994). The focus of this review is on processes occurring in this bright central region of our Galaxy. In this section we outline the unique properties of the interstellar medium (ISM) in the CMZ and examine the links between its different components.

\subsection{The Central Molecular Zone}

The structure of our Galaxy's CMZ is best delineated by high-resolution observations of the CO molecule (Bania 1977; Liszt \& Burton 1978; Heiligman 1987; Bally et al 1987, 1988; Oka et al 1996). At galactocentric radii in excess of a few hundred parsecs, the emissivity of CO is low (Scoville \& Sanders 1987), reflecting an average molecular surface density $\sim 5 M_{\odot} \mathrm{pc}^{-2}$ in the H I nuclear disk (Burton 1992, Burton \& Liszt 1993, Boyce \& Cohen 1994, Liszt 1996, and references therein), a mixed molecular/atomic (and markedly tilted) layer that occupies the region inside of our Galaxy's "4-kpc molecular ring." However, at a radius of roughly $200 \mathrm{pc}$, a transition occurs to a largely molecular, high-density $\left(n \gtrsim 10^{4} \mathrm{~cm}^{-3}\right)$, high volume filling factor $(f \gtrsim 0.1)$ medium containing 5-10 $\times 10^{7} M_{\odot}$ of gas (Armstrong \& Barrett 1985; Walmsley et al 1986; Bally et al 1987, 1988; Güsten 1989; Stark et al 1989; Tsuboi et al 1989). Such densities are usually found only in molecular cloud cores, with small net filling factor, but in the CMZ, stable clouds require such densities to withstand tidal shearing (Güsten \& Downes 1980). However, although high densities are required, the high surface density (several hundred $M_{\odot} \mathrm{pc}^{-2}$ ) and total mass content are not, suggesting mass concentration due to inflow from larger radii.

The clouds in the CMZ also show significantly elevated temperatures (30-200 K, typically $70 \mathrm{~K}$; Güsten et al 1981, Morris et al 1983, Armstrong \& Barrett 1985, Mauersberger et al 1986, Hüttemeister et al 1993). Such gas temperatures are high in comparison both to outer Galaxy clouds and to coextensive dust, calling for a direct and widespread gas heating mechanism. Highly supersonic internal velocity dispersions $\left(\sim 15-50 \mathrm{~km} \mathrm{~s}^{-1}\right)$, comparable to the intercloud velocity dispersion, are also the rule. The elevated temperatures and linewidths may be linked, as dissipation of turbulent energy is a prime candidate for direct 


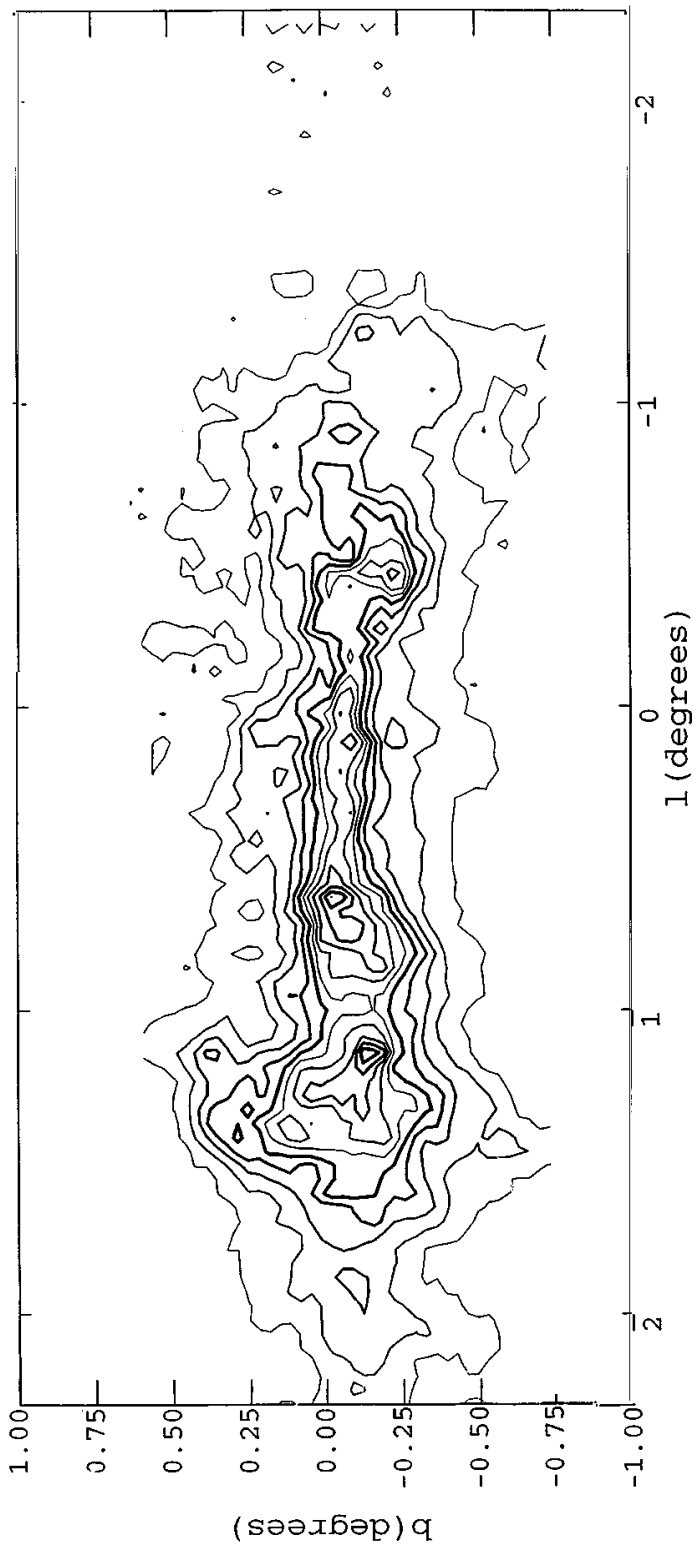




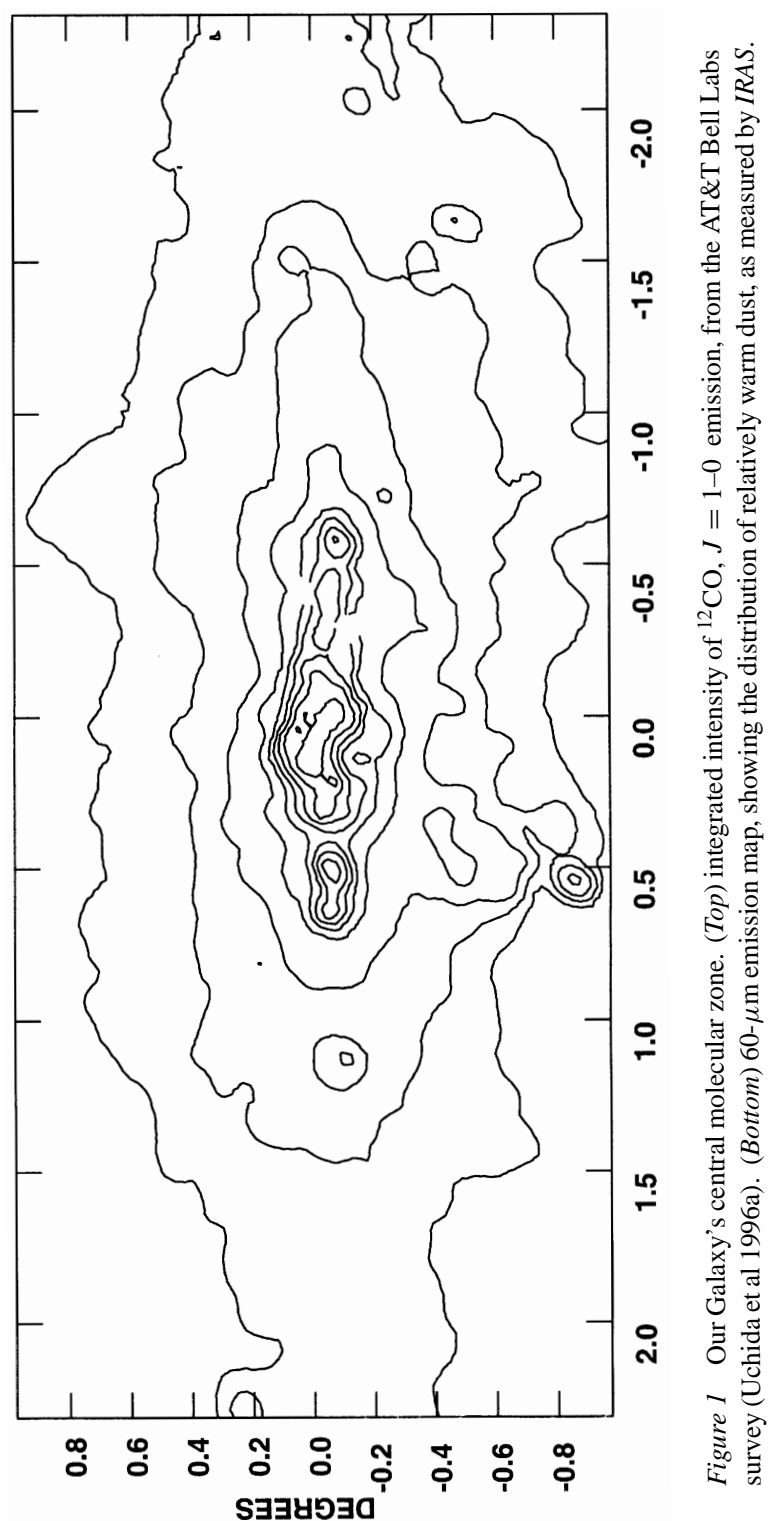


heating of the gas (Wilson et al 1982). This remains true if the cloud velocity dispersions are due to magnetosonic disturbances (Section 2.5). Although energy extraction from the tidal field has been proposed as the origin of the large linewidths (Fleck 1980), Das \& Jog (1995) conclude that this mechanism is efficient only for a central bar potential, in which tidal fields vary markedly along elongated orbits. Viscous magnetic heating resulting from the drift of ions and grains through the neutral gas (ambipolar diffusion) also warrants strong consideration in this highly magnetized environment (Roberge et al 1995).

The distribution and kinematics of the gas in the $\mathrm{CMZ}$ are inconsistent with both axial symmetry and uniform circular rotation (Bania 1977, Liszt \& Burton 1978, Morris et al 1983, Heiligman 1987, Bally et al 1988, Jackson et al 1996). The distribution is such that roughly three quarters of the dense molecular gas is located at positive longitudes, three quarters is at positive velocities, and large radial and vertical motions are present in a significant fraction $(30 \%)$ of the gas (Bally et al 1988). On the basis of molecular kinematics, it is possible to divide the CMZ gas into two components. The first is a high-velocity (130-200 $\mathrm{km} \mathrm{s}^{-1}$ ), quasi-continuous ring structure surrounding the nucleus and having a radius ( $\sim 180 \mathrm{pc}$ ) suggesting a location at the outer boundary of the CMZ. The kinematics of this ring (hereafter referred to as the "180-pc molecular ring") and its tilt relative to the Galactic plane are continuous with those of the exterior $\mathrm{H}$ I nuclear disk, indicating that this structure likely marks the location of an $\mathrm{H}$ $\mathrm{I} / \mathrm{H}_{2}$ transition (Binney et al 1991).

Inside of this "boundary" lies the second, mass-dominant molecular component: a lower-velocity (typically $\lesssim 100 \mathrm{~km} \mathrm{~s}^{-1}$ ) population of dense and massive molecular clouds. This cloud population (which includes the familiar clouds associated with the Sgr A-Sgr E H II regions) lies quite close to the true Galactic plane and is referred to as the Galactic center "disk population" (Bally et al 1988, Heiligman 1987). This disk population contains filament-like clouds with coherent velocity gradients over scales of 30-100 pc, suggestive of dust lanes and tidally stretched arcs or arms of gas (Stark \& Bania 1986; Serabyn \& Güsten 1987; Bally et al 1988; Sofue 1995a,b). Several of these extended clouds contain a large fraction of the total molecular mass (Bally et al 1988, Güsten 1989). Sgr B2, the most massive molecular cloud in the Galaxy, contains about $5 \%$ of the gaseous mass present in the $\mathrm{CMZ}$, while a handful of large clouds contributes up to one third of the CMZ's CO line flux. Because of our vantage point in the disk of the Galaxy, the true geometric arrangement of these clouds and structures remains ambiguous, but a framework for interpretation appears possible in the context of gas flows in a barred potential (Section 3).

One of these massive molecular clouds, the $50 \mathrm{~km} \mathrm{~s}^{-1}$ cloud, is adjacent to the Galactic center in projection. It is associated with the bright, central 
Sgr A complex (Ekers et al 1983, Yusef-Zadeh \& Morris 1987a, Pedlar et al 1989), which itself consists of three components: (a) the Sagittarius A West $\mathrm{H}$ II region and which is centered more or less on the central stellar cluster; (b) the somewhat larger-scale nonthermal shell source Sgr A East, which is offset from the center, but which encloses Sgr A West in projection (Section 5 provides further detail); and (c) the compact, central radio source Sgr A* (Section 6).

The distributions of both ionized gas and diffuse infrared emission in the CMZ (Altenhoff et al 1978, Odenwald \& Fazio 1984, Handa et al 1987, Cox \& Laureijs 1989) are more symmetric than the CO distribution (Figure 1), suggesting that heating sources are more evenly distributed than are the discrete clouds. In particular, the dust temperature drops regularly as $r^{-0.3}$ from its central peak to its asymptotic value of $23 \mathrm{~K}$ near a radius of $200 \mathrm{pc}$. The Lyman continuum production rate and far-infrared luminosity of the CMZ are $\sim 1-3 \times 10^{52} \mathrm{~s}^{-1}$ and $10^{9} L_{\odot}$, respectively, and infrared excesses of roughly 10 and 30 are deduced for individual H II regions and the extended emission, respectively (Mezger \& Pauls 1979, Reich et al 1987, Cox \& Laureijs 1989). The former is typical of Galactic H II regions, while the latter requires a later population of stars. Star formation with an underabundance of $O$ stars had been considered (Odenwald \& Fazio 1984, Lis \& Carlstrom 1994), but the abundant population of $\mathrm{K}$ and $\mathrm{M}$ giants in the Galactic center suffices to heat the dust in the extended region to the observed levels (Cox \& Laureijs 1989) without adjusting the $\mathrm{O} / \mathrm{B}$ ratio. A soft interstellar radiation field also receives support from the underabundance of $\mathrm{C}^{+}$emission from the CMZ (Nakagawa et al 1996).

\subsection{The Hot Component}

Extended X-ray emission centered on the Galactic nucleus has also been detected, with a size $\left(1.8^{\circ} \times 1.0^{\circ}\right)$ roughly half that of the CMZ. This region stands out most clearly in the intense 6.7-keV K $\alpha$ transition of He-like Fe (Koyama et al 1989, Yamauchi et al 1990), although it is also seen in the continuum (Kawai et al 1988, Sunyaev et al 1993, Markevitch et al 1993) and in the 6.4$\mathrm{keV} \mathrm{K} \alpha$ transition of neutral Fe (Koyama 1996, Koyama et al 1996). The continuum emission drops rapidly with frequency, and this central region is not seen at energies above $35 \mathrm{keV}$ (Goldwurm et al 1994). Skinner (1989) provides a complete review of earlier X-ray observations; here we concentrate on recent results from the ASCA, Ginga, and GRANAT spacecraft.

The detection of the $6.7-\mathrm{keV}$ line from He-like Fe clearly reveals the existence of a high-temperature plasma in the central hundred or so parsecs. Estimates for its temperature vary, from 10-15 keV (Koyama et al 1989, Yamauchi et al 1990, Nottingham et al 1993) down to 1-3 keV (Markevitch et al 1993). The latter estimate is lower because of the finding that the X-ray emission changes 
character for energies above $\sim 10 \mathrm{keV}$; the lower energy emission shows a roughly elliptical distribution, but the higher energy emission essentially mimics the CMZ's flattened distribution. This, and the image of the neutral $\mathrm{Fe} \mathrm{K} \alpha$ transition, which shows a clear correlation with the dense clouds (Koyama 1996, Koyama et al 1996), strongly suggest that scattering of X-rays by the high-column-density medium in the CMZ is of importance, both in terms of the $6.4-\mathrm{keV}$ line and of the continuum above $10 \mathrm{keV}$. X-ray data thus also provide information on the cold molecular medium, and by inference, on the discrete X-ray sources that illuminate it (Sunyaev et al 1993, Koyama et al 1996).

Although estimates vary, the hot plasma has a temperature of some $10^{7}-10^{8}$ $\mathrm{K}$. The upper end of this range is quite high, more akin to the temperatures found for intergalactic gas bound to clusters of galaxies than to the temperatures found for individual galactic nuclei or supernova remnants (Holt \& McCray 1982, Pietsch 1994, Koyama et al 1996). At temperatures near $10^{8} \mathrm{~K}$, the gas would not be bound to the Galaxy at all (Yamauchi et al 1990, Sunyaev et al 1993), and a wind would expand from the center at a few thousand kilometers per second, implying a lifetime of $\sim 10^{5} \mathrm{yr}$. The energy and mass requirements would then be severe, with the plasma containing some $10^{53} \mathrm{ergs}$ and $\sim 3000 M_{\odot}$ (Yamauchi et al 1990, Koyama et al 1996), at an average density of 0.3-0.4 $\mathrm{cm}^{-3}$. To account for the extreme inferred temperatures, these authors propose an energetic explosion in the Galactic center at a past epoch. However, the necessary prior luminosity, $10^{41}-10^{42} \mathrm{erg} \mathrm{s}^{-1}$, dramatically exceeds anything observed today (Section 6). On the other hand, the lower temperatures implied by removal of the high-energy scattering component from the temperature determination yield plasma temperatures more in line with supernova remnant temperatures, but the emission measure then requires a rather large number of supernovae $(\sim 1000)$. It has thus been difficult to settle on a single scenario for this plasma that does not involve past releases of energy in the Galactic center far exceeding the current rate. Perhaps the simplest explanation would be a past starburst, in which the ensuing supernovae generate the high-temperature plasma.

Scattering of high-energy photons also provides a tracer for past activity through the source-scatterer time delay; from the observed distribution of highenergy X rays, Sunyaev et al (1993) conclude that the X-ray luminosity of the central $\sim 100 \mathrm{pc}$ has not exceeded the current luminosity of $\sim 10^{37} \mathrm{erg} \mathrm{s}^{-1}$ by more than a factor of 10-100 in the past several hundred years, whereas scattering in the $\mathrm{K} \alpha$ line of $\mathrm{Fe}^{\circ}$ leads Koyama et al (1996) to infer that a single source must have brightened to $>10^{39} \mathrm{erg} \mathrm{s}^{-1}$ in roughly this same interval. The $1.8-\mathrm{MeV}$ line of ${ }^{26} \mathrm{Al}$, which traces past massive star formation on a few 


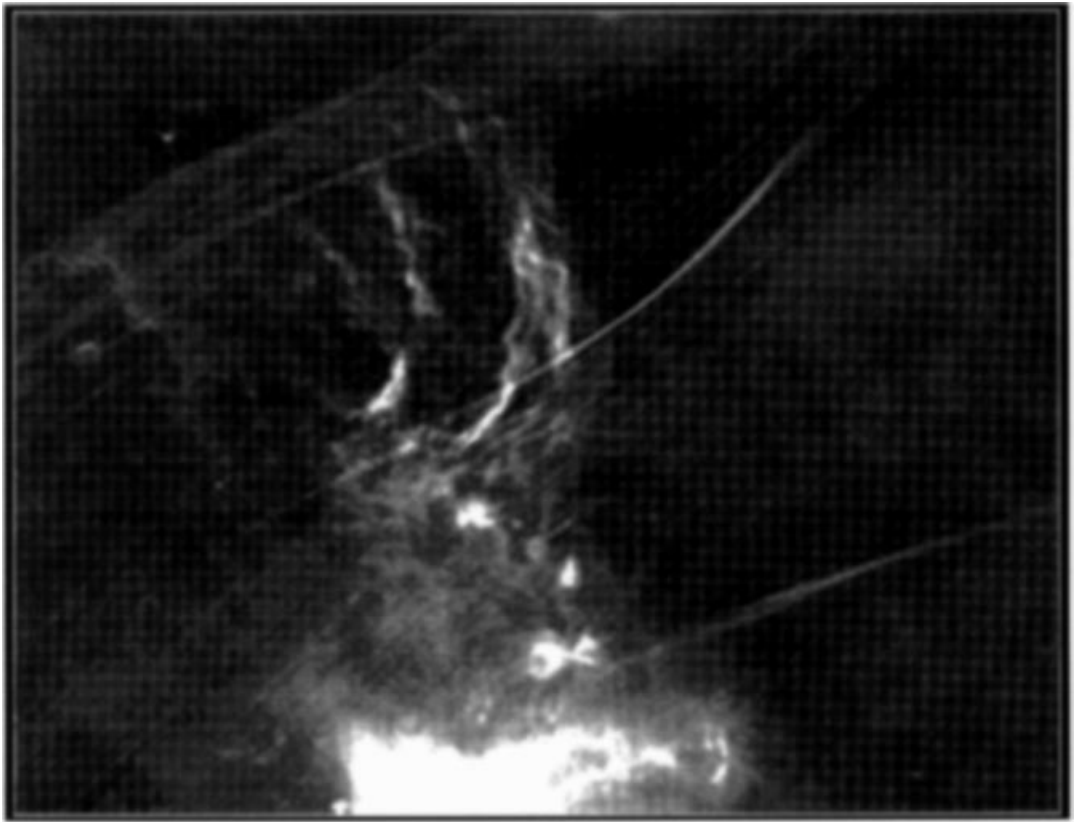

Figure 2 Radiograph of 20-cm emission arising from the Northern and Southern Threads, made with the VLA (Echevarria \& Morris 1996). The vertical scale is 16.5 arcminutes ( $40 \mathrm{pc})$. The Sgr A complex is visible at bottom center. The radio Arc (upper left) appears weak because it is beyond the telescope's primary beam.

million-year time scale, shows a peak near, but offset from, the Galactic Center (Diehl et al 1995), and so it may be relevant in tracing past activity in the central region also. However, a coherent picture of the recent past of our Galactic nucleus has not yet emerged.

\subsection{The Galactic Center Magnetosphere}

The magnetic field within the CMZ probably plays a significant role in the physical interactions occurring there. Its presence is revealed in several ways, the most striking of which is the observation of seven or eight systems of nonthermal radio filaments (NTFs) within $\sim 150 \mathrm{pc}$ of the Galactic center (Yusef-Zadeh 1989; Morris 1990, 1994, 1996). These polarized structures are tens of parsecs long and only a fraction of a parsec wide. They may occur in isolation (in which case they have been called threads; Morris \& Yusef-Zadeh 1985) or in bundles, such as those comprising the linear portion of the prominent Radio Arc (Yusef-Zadeh et al 1984). Morphologically, the NTFs are strikingly uniform 
in brightness and curvature (Figure 2) and therefore quite different from the meandering, contorted filamentary structures one finds in supernova remnants and emission-line nebulae. The morphology of the NTFs suggests that they reflect the local magnetic field direction, and radio polarization evidence supports that notion. Tsuboi et al (1986) corrected the observed polarization angles within the NTFs of the Radio Arc for Faraday rotation and deduced that the intrinsic field is parallel to the filaments, a result confirmed by Reich (1994) and Tsuboi et al (1995) at high frequencies ( 32 and $45 \mathrm{GHz}$, respectively), where Faraday rotation is small.

A clue to the strength of the magnetic field in the NTFs is provided by the near absence of deformation or bending along their lengths. Every NTF that has been sufficiently well studied has been found to be associated with, and is probably interacting with, at least one molecular cloud (detailed below). However, the magnetic filaments are not subject to large distortions at the interaction sites, in spite of the large velocity dispersion within Galactic center molecular clouds, and in spite of the likelihood that, given the large intercloud velocity dispersion at the Galactic center, most clouds have a typical velocity of at least a few tens of kilometers per second with respect to the ambient magnetic field. By equating the apparent turbulent pressures within clouds (or the ram pressure associated with presumed cloud motion relative to the field) to the magnetic pressure, as a minimum condition on the strength of the magnetic field, Yusef-Zadeh \& Morris (1987b,c; 1988) have determined that the magnetic field within the NTFs has milligauss strength. [The one known exception is G359.1-0.2, the Snake filament (Gray et al 1991, 1995), which is endowed with a few "kinks," suggesting that turbulence in its environment has partially overcome the rigidity of the field. However, this filament is the one with the greatest projected distance from the Galactic nucleus (125 pc), so it may imply a declining field strength within filaments at larger distances.] This "rigidity" method of constraining the magnetic field strength in NTFs needs to be recast if the clouds consist of a number of independently moving clumps (cf Serabyn \& Morris 1994), because the field lines can then be deflected around and between the clumps as the ensemble of clumps moves through the field. A modified picture of this sort does not dramatically change the constraint on the magnetic field strength; the time scale to transport clump-induced distortions of the interclump field lines out of the cloud by Alfvén waves, $2 \times 10^{4} B_{\mathrm{mG}}^{-1}\left(n / 10 \mathrm{~cm}^{-3}\right)^{0.5}(D / 10 \mathrm{pc}) \mathrm{yr}$ for cloud size $D$ and interclump density $n$, must be less than a clump crossing time (typically $2 \times 10^{4}$ years), which still implies $\gtrsim m G$ field strengths.

The fact that all of the known NTFs are perpendicular to the Galactic plane to within about $20^{\circ}$ (Morris \& Yusef-Zadeh 1985, Anantharamaiah et al 1991) suggests that these structures trace an ubiquitous dipole magnetic field occurring 
on a scale comparable to that of the CMZ. In this view, the observed filaments are the magnetic flux tubes that happen to be illuminated by the local injection of relativistic particles. A few alternative hypotheses have been considered, however. One - that the site of each NTF or NTF bundle represents a locally generated field enhancement-encounters difficulties because of the strength of the magnetic field in the NTFs. If the milligauss fields are present only in the NTFs, then a strong confinement mechanism operating along their full $\sim 30$-pc length is needed to prevent them from expanding at the Alfvén speed and thus disappearing on a time scale of $\sim 10^{3} \mathrm{yr}$. This is much faster than is needed to establish the currents necessary to generate the structure in the first place (comparable to a dynamical crossing time of $\gtrsim$ a few times $10^{4} \mathrm{yr}$ ) and is shorter than the energy-loss time scale for relativistic electrons in the filaments (Anantharamaiah et al 1991, Sofue et al 1992). Another alternative is that the NTFs lie upon the surface of a cylindrical wall of compressed magnetic field surrounding the Galactic center (Uchida et al 1985, Heyvaerts et al 1988) and that they are again illuminated by some local mechanism for the generation of relativistic particles. Such a geometry could result from expansive gas motions from the center (Umemura et al 1988), if the gas momentum were sufficiently large, although the expansive events would have to be continuous, quite recent, or even in progress (such as would be manifested by the hypothetical, expanding molecular shell described in Section 3.1). This geometry cannot be ruled out, although it would imply a remarkable pattern of currents that might be difficult to maintain.

A twisted poloidal magnetic field geometry has been considered for the Galactic center, on the assumption that this would result from flux-freezing of field lines into the differentially rotating disk gas (Uchida et al 1985, Sofue et al 1987, Shibata \& Uchida 1987). However, the evidence for such a geometry is based on rotation measures, which are difficult to localize to the Galactic center and which only probe the presumably relatively weak, line-of-sight magnetic field component. The more direct indicators of the dominant field geometrythe NTFs - pass through the gas layer in the Galactic disk without showing any appreciable large-scale twist or distortion, other than perhaps a slow divergence. A large-scale twist might be hidden by a fortuitous projection of one or two filaments, but it is unlikely that all seven or eight known NTF systems would be similarly projected. The apparent immunity of the NTFs to the inertia of the disk gas could be understood if the fields within molecular clouds are only weakly coupled to the external field (i.e. separated by magnetopauses).

2.4.1 MID AND FAR-INFRARED POLARIZATION The second most informative probe of the magnetic field near the Galactic center has been the polarization of thermal infrared emission from dust. Various mechanisms have been 
proposed for orienting the emitting dust grains in such a way that their spin axes are preferentially aligned along the magnetic field (Hildebrand 1988, Lazarian 1994, Roberge et al 1995). All potentially viable mechanisms imply the same relationship — orthogonality - between the polarization position angle and the projected magnetic field direction. A map of the distribution of polarization position angles is thus readily transformed into a map of the projection of the magnetic field onto the plane of the sky, weighted by the volume emissivity of the dust along the line of sight. The dust emissivity is maximized in dense clouds or at warm cloud surfaces, so in contrast to the NTFs, which depict the field geometry in the intercloud medium, far-IR polarization tends to probe the magnetic field within dense clouds. The gross uncertainties about the specifics of the grain alignment mechanisms make quantitative links between the field strength and the fractional polarization difficult, although the magnitude of field direction fluctuations can be indicative of the field strength (Morris et al 1992, 1996a,b).

Polarization measurements were first applied to the Galactic center by Aitken et al $(1986,1991)$, who, observing at $10 \mu \mathrm{m}$, found that the magnetic field within the northern arm of Sgr A West (cf Section 5) is parallel to that arm, a result that would arise naturally from the action of shear within this orbiting gas stream. Shear is expected to affect much of the gas present within the strong tidal field of the Galactic center, so this result may have widespread applicability. Strong far-IR polarization has now been mapped in four clouds near the Galactic center, as reviewed by Davidson (1996). In essentially all of the clouds, the inferred magnetic field direction is predominantly parallel to the Galactic plane (Sgr B2 is a complex mix of absorption and emission and is still ambiguous in this regard), which stands in marked contrast to the perpendicular field inferred for the intercloud medium. Figure 3 shows the $60-\mu \mathrm{m}$ polarization vectors toward G0.18-0.04 (Morris et al 1996b), superimposed upon a 6-cm radiograph (YusefZadeh \& Morris 1987b) and the underlying cloud (Serabyn \& Güsten 1991). The implied field direction within the cloud is parallel to the ridge of molecular and thermal radio emission, which is largely parallel to the Galactic plane. The implication that the magnetic field follows the ridge of the molecular cloud in this and in the nearby arched filament cloud suggests that the field within clouds is shaped by the stresses that shape the clouds themselves (Serabyn \& Güsten 1987; Morris et al 1992, 1995). Furthermore, the general orthogonality of fields inside and outside of clouds suggests that the two are relatively independent, consistent with the apparent immunity of NTFs to the inertia of disk gas.

The shaping of the fields within clouds by the stresses which shape clouds themselves appears to apply to the circumnuclear disk (CND) as well (Section 5; Werner et al 1988; Hildebrand et al 1990, 1993). With the exception of a 


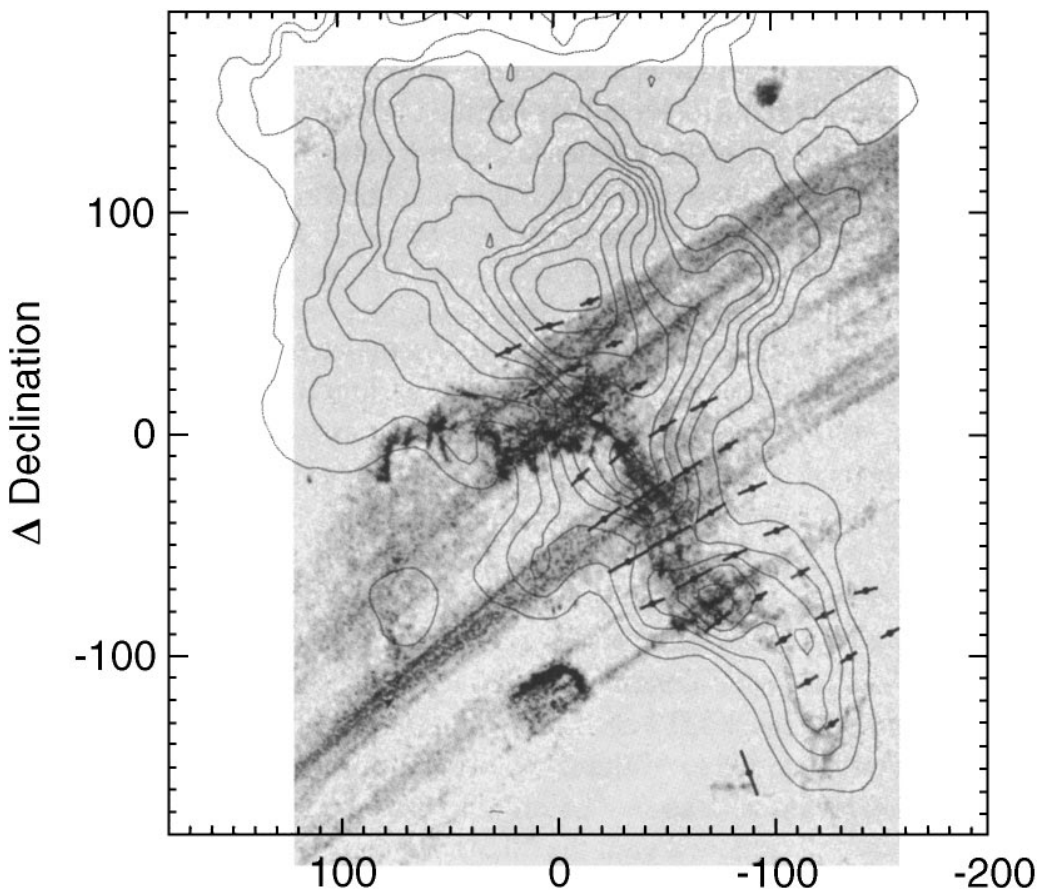

$\Delta$ Right Ascension

Figure $3 \quad 60-\mu \mathrm{m}$ polarization measurements across the H II region G0.18-0.04 (from Morris et al 1996b), superimposed upon a $\lambda 6$-cm continuum radiograph (from Yusef-Zadeh \& Morris 1987b). The orientations of the line segments represent the position angles of the electric vectors; their length is proportional to the percent polarization. A horizontal segment representing $4 \%$ polarization is shown at bottom left. The position angle of the Galactic plane is $\sim 30^{\circ}$, so the polarization vectors are all close to being perpendicular to the Galactic plane. The superimposed contours show the intensity of CS $J=3-2$ emission, from Serabyn \& Güsten (1991). 
presumably infalling stream of dust and gas coinciding with the Northern Arm of Sgr A West, all of the far-IR emission from dust in the CND is polarized in a manner consistent with a dominant magnetic field component parallel to the plane of the disk. Self-similar models for a poloidal field well outside the disk (i.e. perpendicular to the disk plane), which has been deformed and sheared within the CND by a combination of differential rotation and radial infall, were proposed by Wardle \& Königl (1990). Variations of these models have generally been rather successful in accounting for the polarimetric mapping results on this source (Hildebrand et al 1993). Another model based on numerical magnetohydrodynamic calculations carried out by Meglicki et al (1994) is also able to reproduce the gross geometric and magnetic characteristics of the CND, although this model disk is undergoing rapid evolution, with inward radial motions $\left(\sim 50 \mathrm{~km} \mathrm{~s}^{-1}\right)$ well in excess of the observational limits $\left(\sim 20 \mathrm{~km} \mathrm{~s}^{-1}\right.$, cf Section 5).

2.4.2 THEZEEMANEFFECT Although the Zeeman effect has been used to probe the magnetic field in Galactic center clouds directly, the results are mixed. The large velocity breadth of the lines from essentially all Galactic center clouds have made Zeeman measurements difficult for all but the strongest fields. Using H I absorption, Schwarz \& Lasenby (1990) derived a modest line-ofsight field, $B_{\|}=0.5 \mathrm{mG}$, toward the northern side of the CND. With 1667$\mathrm{MHz} \mathrm{OH}$ absorption, Killeen et al (1992) measured $B_{\|}=-2.0 \mathrm{mG}$, averaged separately over broad portions of the northern and southern sides of the CND, with considerable spatial variation in $B_{\|}$on scales of 5 to $10^{\prime \prime}$. Most recently, Plante et al (1995) used $\mathrm{H}$ I absorption to detect fields ranging up to about -3 $\mathrm{mG}$ on the northern side of the CND. They attribute the strongest fields to a feature interpreted as a stream of infalling gas rather than a part of the disk. These results, all obtained with the VLA, and the negative $\mathrm{H}$ I Zeeman results of Marshall et al (1995), who obtained $B_{\|}<0.5 \mathrm{mG}$ in $45 \times 45 \operatorname{arcsec}^{2}$ regions of the CND, suggest that the average line-of-sight component of the field within the CND is $\sim 1 \mathrm{mG}$, but the likelihood that $B_{\|}$changes considerably and even undergoes reversals along the line of sight (for example, in the model of Wardle \& Königl 1990) implies that the Zeeman signals are diminished by projection effects.

Elsewhere in the Galactic center, Zeeman measurements have yielded negative results. Uchida \& Güsten (1995) observed OH absorption in a number of clouds with a relatively large beam; they obtained typical upper limits to $B_{||}$of a few tenths of a milliGauss. Although their observations are subject to dilution of the Zeeman signal by averaging over spatial variations within the beam, as well as along the line of sight, these results raise questions about the conclusion that milligauss fields pervade the Galactic center region, unless either the line- 
of-sight component is small everywhere (i.e. a field that is everywhere within $\sim 10^{\circ}$ from the vertical to the Galactic plane) or the field has strong spatial variations.

2.4.3 FARADAY ROTATION MEASURES Faraday rotation measures (RMs) provide yet another probe of the field surrounding nonthermally emitting Galactic center radio structures. Vast areas of the Galactic center display polarized emission (Sofue et al 1987, Haynes et al 1992), so in principle the magnetic field within a large volume of the intervening medium can be studied, although this probe has not yet been extensively utilized. The RMs can be quite large at some sites within the Galactic center. Using 6-cm radio observations, Yusef-Zadeh \& Morris $(1987 \mathrm{c}, 1988)$ report RMs up to 5500 radians $\mathrm{m}^{-2}$ toward the Radio

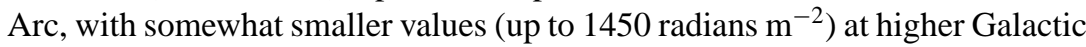
latitudes (see also Tsuboi et al 1995). In a 2-cm study of the Radio Arc (Inoue et al 1989), complex structure was seen in the foreground Faraday screen, even to the extent that depolarizing filaments were identified, but these have no obvious counterpart in total intensity images. When this kind of study is performed more thoroughly and quantitatively, with independent measures of electron density, it will provide much-needed information on the field strength and geometry of magnetic structures.

2.4.4 THE PRODUCTION OF RADIO FILAMENTS A number of hypotheses about the origin of the Galactic center NTFs have been offered (Chudnovsky et al 1986, Heyvaerts et al 1988, Benford 1988, Morris \& Yusef-Zadeh 1989, Serabyn \& Güsten 1991, Lesch \& Reich 1992, Rosso \& Pelletier 1993, Serabyn \& Morris 1994), the merits and drawbacks of which are summarized by Morris (1996). Here, we describe only the hypothesis that, in our judgement, conforms best to the observational evidence. The key to this hypothesis is that all sufficiently well-studied NTFs appear to be interacting with molecular structures. It was originally suggested following examination of molecular CS emission from the cloud underlying the G0.18-0.04 H II region (Serabyn \& Güsten 1991, Serabyn \& Morris 1994; see also Morris 1995, Serabyn 1995). Yusef-Zadeh \& Morris (1987b) had pointed out that most of the nonthermal filaments of the Galactic Center Radio Arc undergo abrupt discontinuities where they encounter the ionized ridge of G0.18-0.04, and Serabyn \& Güsten (1991) demonstrated that this H II region lies at the surface of a relatively massive molecular cloud. With a partial interferometric view of the molecular cloud, we found that dense clumps are present at a number of the locations where the NTFs undergo brightness discontinuities or small deflections (Serabyn \& Morris 1994). This led us to propose that the NTFs in the Radio Arc, and perhaps all Galactic center threads, originate at the surfaces of such dense molecular clumps. The hypothesis can 
be stated as follows: When certain conditions are satisfied by a molecular cloud moving through a more diffuse ambient medium, magnetic field line reconnection taking place at leading clump surfaces leads to the acceleration of charged particles, and the resultant relativistic particles then stream away from their point of origin along the field lines. The flux lines to which these particles are attached are then illuminated by synchrotron emission.

The hypothesized conditions for particle acceleration are: (a) The cloud surface must be ionized, presumably by a fortuitously placed local source of ionizing radiation. The ionized gas provides a source of free electrons for the acceleration mechanism to act upon, and the turbulence associated with the ionization front ensures that the cloud and intercloud magnetic fields are sufficiently mixed for reconnection to be efficient. (b) The cloud surface at which the acceleration takes place must be moving at a relatively large velocity into the ambient intercloud medium, so as to force the magnetic fields in the two media into contact. $(c)$ The orientations of the fields in the cloud and intercloud media must be quite different for rapid energy extraction, a condition that has very recently been verified in the case of G0.18-0.04, where far-IR polarization measurements show that the internal cloud field is perpendicular to the linear radio filaments with which the cloud is interacting (Figure 3). The same may be true for a substantial fraction of all Galactic center clouds, if the trends from far-IR polarimetry are any guide. However, the conditions listed here are collectively stringent enough to explain why it is that not every molecular clump in the Galactic center magnetosphere has an associated NTF.

Conversely, it is possible that these conditions are satisfied by every filament or thread in the Galactic center. The NTFs associated with Sgr C arise (in projection) at the interface between that bright $\mathrm{H}$ II region and its associated molecular cloud (Liszt \& Spiker 1995); the filament G359.5+0.18, located to the north of $\mathrm{Sgr} \mathrm{C}$, is apparently interacting with at least one, and possibly two, molecular clouds (Bally et al 1989, Staguhn et al 1996); the Northern thread (G0.08+0.15; Morris \& Yusef-Zadeh 1985) undergoes an abrupt intensity discontinuity where it is superimposed upon the thermal arched filament $\mathrm{H}$ II region and associated cloud (Figure 2); and finally, an H II region/molecular cloud complex is located at the northern tip of the Snake (G359.1-0.2; Gray et al 1995, Uchida et al 1996b). However, in none of these cases is a cause-and-effect relationship between an NTF and a molecular cloud/H II region as convincing as the Radio Arc case because high-resolution observations are lacking.

2.4.5 ORIGIN OF THE STRONG POLOIDAL FIELD If the Galactic center magnetosphere is pervasive on a scale of $\sim 100 \mathrm{pc}$, then its total energy content is $\sim 10^{54}\langle B(\mathrm{mG})\rangle$ ergs, comparable to the energy content of the hot, X-ray emitting gas and only a few times larger than the kinetic energy associated with 
noncircular motions in the 180-pc molecular ring (Section 3). The maintenance of such a strong, dynamically important field requires a rather powerful ring current circulating about the center, presumably somewhere within, or at the boundary of, the CMZ. The maintenance of such a current, and the confinement of the field, might be accomplished by the Lorentz forces accompanying the quasi-steady radial inflow or outflow (or both) of gas through a vertical magnetic field (e.g. Lesch et al 1989), although the physical elements of such a dynamo mechanism still require elaboration.

One hypothesis for the vertical field at the Galactic center, which is appealing both for its simplicity and its seeming inevitability, is that of Sofue \& Fujimoto (1987). Noting that Galactic evolution is characterized by the inexorable radial inflow of matter, these authors hypothesized that the vertical component of the early Galactic field is dragged inwards by the gas accreting to the Galactic center region over the lifetime of the galaxy; its rate of outward radial diffusion with respect to the gas is small compared to the rate of inward gas flow, thereby concentrating the vertical field at the nucleus. In contrast, the component of the field parallel to the disk can be transported vertically out of the Galaxy by diffusion or by the Parker instability on a time scale that is short compared to a Hubble time, so that the azimuthal field represents an equilibrium between the flux lost by vertical transport and that regenerated by a Galactic dynamo.

According to the Sofue \& Fujimoto hypothesis, the strength of the vertical field concentrated at the nucleus reflects the strength of the primordial magnetic field. However, it also depends on the spectrum of spatial fluctuations, since the only fluctuations that could survive to form a uniform field when concentrated at the nucleus are those present initially on Galactic scales or larger (Morris 1994). Smaller-scale field fluctuations would have suffered reconnection and annihilation in the Galactic center mix. These processes, along with the conversion of gravitational potential energy of the inflowing gas, may have been important sources of heating for Galactic center gas throughout Galactic history.

\subsection{Pressure Balance}

The physical conditions present in the CMZ are consistent with thermal, nonthermal, and magnetic pressures several orders of magnitude higher than those present in the large-scale Galactic disk. For molecular densities of $10^{4} \mathrm{~cm}^{-3}$ at the inferred temperatures (Section 2.2), the pressure $P_{\text {thermal }} \sim 10^{-10} \mathrm{erg}$ $\mathrm{cm}^{-3}$. In the hot plasma, $P_{\mathrm{hot}} \sim 4 \times 10^{-10} \mathrm{erg} \mathrm{cm}^{-3}$, close enough to consider pressure balance (Spergel \& Blitz 1992). However, turbulent pressures in the clouds greatly exceed this value, approaching $P_{\text {turb }} \sim 10^{-8} \mathrm{erg} \mathrm{cm}^{-3}$. For a field strength of $0.1-1 \mathrm{mG}$, the magnetic pressure is comparably large: $P_{\text {mag }} \sim 4 \times 10^{-10}$ to $4 \times 10^{-8} \mathrm{erg} \mathrm{cm}^{-3}$. Internal cloud turbulence is therefore 
likely to be linked to magnetic pressures. In particular, the observed velocity dispersions in Galactic center clouds likely reflect hydromagnetic waves (Arons \& Max 1975), which could be generated by the angular momentum loss processes affecting Galactic center clouds (described in Section 3), including shocks, cloud collisions, and the irregular viscous interactions suffered by clouds moving through a magnetized, low-density medium.

\section{THE CENTRAL BAR; GAS DYNAMICS NEAR THE GALACTIC CENTER}

The presence of a substantial stellar bar in our Galaxy, first invoked to account for the noncircular motions of H I near the Galactic center (de Vaucouleurs 1964, Peters 1975), is now well established by a variety of methods, including techniques based on photometry (Blitz \& Spergel 1991b, Sellwood 1993, Weiland et al 1994, Dwek et al 1995), kinematic studies of gas (Liszt \& Burton 1980, Mulder \& Liem 1986, Binney et al 1991, Wada et al 1994) and stars (Zhao et al 1994, Blum 1995), and counts of luminous stars (Nakada et al 1991; Weinberg 1992a,b; Whitelock \& Catchpole 1992; Stanek et al 1994). The picture that has emerged is as follows: The bar is quite pronounced, with axial ratios of about 3:1:1 [though Binney et al (1991) and Wada et al (1994) prefer 1.5:1 for the axial ratio in the Galactic plane]; it extends at least to the corotation radius of about $2.4 \mathrm{kpc}$; it has a total mass of $1-3 \times 10^{10} M_{\odot}$; and its long axis is oriented at a modest angle with respect to our line of sight (15 to $45^{\circ}$ toward positive longitude, depending on the model).

The excess of microlensing events toward the Galactic bulge provides yet another indication that the Galactic bulge is strongly barred and that the bar is somewhat along our line of sight (Paczyński et al 1994, Han \& Gould 1995, Zhao et al 1995). Leaving aside the details of these determinations, we turn to the issue of the interstellar material that coexists with the stellar bar. A strong bar is an essential element for understanding gas dynamics near the Galactic center and especially for appreciating the potential importance of bar-induced migration of interstellar gas toward the Galactic nucleus.

\subsection{Response of Interstellar Gas to a Bar Potential}

The response of orbiting gas to such a pronounced bar is quite strong and leads both to large deviations from circular motion and to strong shocks. Thus, when studying gas dynamics, it is essential to properly account for the $m=2$ (quadrupole) deviation from axial symmetry represented by the bar (where the gravitational potential depends on azimuthal angle, $\phi$, via the factor $e^{i m \phi}$ ). The velocity field of the gas provides one of the best ways of probing the shape of the Galactic gravitational potential. The primary data to be explained by any bar model comprise the distribution of molecular emission (or absorption) 
throughout the longitude-latitude-velocity $(l, b, v)$ cube, and the most remarkable feature of this distribution in our Galaxy is the 180-pc molecular ring (e.g. Bania 1977, Bally et al 1987), seen as a parallelogram, or ellipse, in projections of this cube onto the $l-v$ plane (Figure 4). Models for the response of orbiting gas to a symmetric $m=2$ bar potential (described more fully below) have met with considerable success in accounting qualitatively for the gas kinematics measured in both H I and CO surveys (Binney et al 1991), notably including this parallelogram, and it is now becoming the standard paradigm for Galactic center kinematics, although a quantitatively faithful model is still being sought (e.g. Binney 1994, Jenkins \& Binney 1994). Most importantly, existing models readily account for the abundance of gas near the Galactic center having "forbidden" radial velocities (i.e. with sign opposite to that of Galactic rotation).

3.1.1 ORBITS OF GAS CLOUDS IN THE PRESENCE OF A STRONG BAR Gas moving in response to a bar potential tends to settle into closed, elongated orbits because cloud collisions and consequent energy dissipation act to enforce conformity. Angular momentum loss by the orbiting gas resulting from the processes described more fully in Section 3.2 causes it to drift inward along a family of nested, closed orbits. When the gas is orbiting at radii between that of corotation and the inner Lindblad resonance (ILR) of the bar pattern, it moves on the so-called $X_{1}$ orbits (Contopoulos \& Mertzanides 1977), which are elongated oval orbits that have their major axes aligned with the bar (Figure 5).

As the ILR is approached, however, there is an innermost stable $\mathrm{X}_{1}$ orbit inside of which these orbits become self-intersecting or cusped. Any angular momentum loss by gas in the innermost stable $\mathrm{X}_{1}$ orbit leads to orbit crossings and shocks. The shocks, in turn, imply a further, abrupt loss of angular momentum, which causes the gas to fall inward until it settles onto a new family of closed, elongated orbits lying deeper in the potential well: the $\mathrm{X}_{2}$ orbits. These oval orbits have their long axes oriented perpendicular to the bar. At their apocenter, the outermost $\mathrm{X}_{2}$ orbits are thought to graze the pericenter of the innermost $X_{1}$ orbits. Thus, angular momentum loss by gas in the innermost $\mathrm{X}_{1}$ orbit, for whatever reason, be it via viscous transport of angular momentum outward or by orbit crossings near the cusps, would lead it to collide with gas in the $\mathrm{X}_{2}$ orbits, presumably creating a spray, which leads to a shock at the far side of the innermost $X_{1}$ orbit (Binney et al 1991, Athanassoula 1992, Jenkins \& Binney 1994, Gerhard 1996).

Binney et al (1991) hypothesize that the 180-pc molecular ring corresponds to the innermost stable $\mathrm{X}_{1}$ orbit. The small radial extent of this feature is responsible for the narrowness of the trace of emission defining the parallelogram and for its well-defined, sharp vertices. The shocks along the inside edge of the $X_{1}$ orbits are held responsible for compressing the gas into molecular form, which explains why predominantly molecular gas occupies only the 


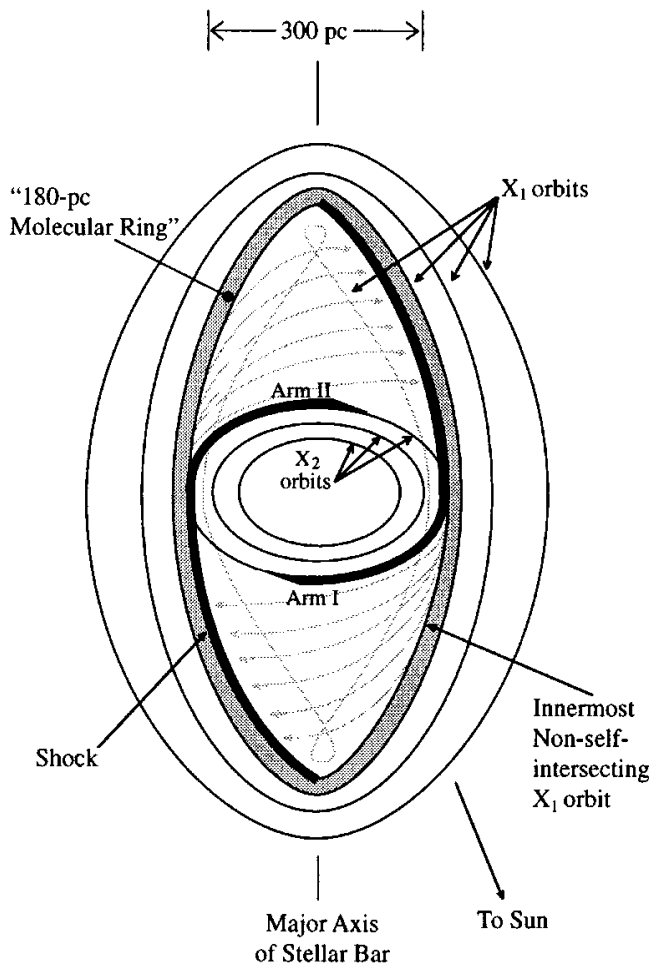

Figure 5 Schematic diagram illustrating the shapes and relative orientations of the $\mathrm{X}_{1}$ and $\mathrm{X}_{2}$ orbits and the locations of the shocks resulting from the interaction between the innermost $\mathrm{X}_{1}$ orbit and the outermost $X_{2}$ orbit. The arms in the CMZ hypothesized by Sofue (1995a) are also shown.

innermost $\mathrm{X}_{1}$ orbits. Indeed, gas located outside the 180-pc molecular ring is evident in H I data (Burton \& Liszt 1978), and the dynamics of this H I nuclear disk can be modeled in terms of a hierarchy of $\mathrm{X}_{1}$ bar orbits (Liszt \& Burton 1980, Binney et al 1991). One potential problem with this interpretation of the parallelogram is the velocities of the vertices (Uchida 1993, Binney 1994): Although the velocity of the negative-longitude, intermediate-velocity vertex is near zero in the models, measurements show it to be large $\left(\sim 150 \mathrm{~km} \mathrm{~s}^{-1}\right)$ and in the forbidden quadrant (Figure 4).

Recall that the 180-pc molecular ring surrounds a complex, asymmetric concentration of disk population molecular clouds. Binney et al (1991) propose that these clouds are distributed on $\mathrm{X}_{2}$ orbits. An attempt to identify some spatial order in this complex distribution was recently made by Sofue (1995a; 
see also Scoville et al 1974), who defines two "arms" of molecular material, seen as continuous streams in the $(l, b, v)$ data cube of ${ }^{13} \mathrm{CO}$ emission [Figure 3 of Liszt (1992) nicely shows the intersection of these streams with the $b=-3^{\prime}$ plane] as the repository of most of the molecular mass interior to the 180 -pc ring. These hypothetical arms have small pitch angles and are roughly symmetrically placed about the Galactic center at radii of $\sim 120 \mathrm{pc}$. Their proposed morphology is similar to the innermost, spiral density enhancements resulting from shocks in a numerical model of gas response to a bar (Gerhard 1996, Athanassoula 1992). It is also similar to the molecular distribution seen in several nearby galaxies (Kenney et al 1992, Turner et al 1993), so the molecular arms may have a natural explanation in the context of bar-induced kinematics. These shocks result from the transition from $\mathrm{X}_{1}$ to $\mathrm{X}_{2}$ orbits, as discussed above, and they should presumably extend continuously out to the 180 -pc molecular ring, although $l-v$ plots show no clear continuity between the arms and the ring.

3.1.2 THE EMR VERSUS THE BAR Perceived in early molecular data as an ellipse in the $l-v$ plane, the $180-p c$ molecular ring was originally interpreted as a radially expanding molecular ring surrounding the Galactic center (Scoville 1972, Kaifu et al 1972) and was subsequently referred to as the EMR. It was hypothesized to be the result of an explosive event at the center $\sim 10^{6}$ years ago, which gave a radial momentum impulse to $\sim 10^{7} M_{\odot}$ of nearby gas (Bania 1977). This hypothesis requires a rather extreme energy $\left(>10^{55}\right.$ ergs when dissipation, work against the gravitational potential, and the nonspherical geometry of the ring are taken into account; Sanders 1989, Saito 1990), although such energies can, in principle, be generated by episodic accretion events at the Galactic center (e.g. Bottema \& Sanders 1986). Another challenge for the EMR hypothesis has been the dearth of evidence that non-EMR clouds in the CMZ have been affected by the passage of this putative flash flood of radially moving material. However, Uchida et al $(1994 a, b)$ recently pointed out that a molecular structure associated with the far-infrared source AFGL 5376 (at $l, b=359.5,+0.43)$ is apparently the site of a large-scale (100-pc) shock oriented perpendicular to the Galactic plane. They argue that it and another vertical complex at $l=1 .{ }^{\circ} 2$ could be the signatures of the interaction of the EMR with the ambient medium. The projection of these shock structures out of the Galactic plane is attributable in part to the tilt $\left(\sim 20^{\circ}\right)$ of the EMR with respect to the Galactic plane. Uchida et al (1994a) also cautioned against abandoning the EMR hypothesis by noting that, when $\mathrm{CO}$ emission within $0 .^{\circ} 15$ of the Galactic plane is excluded from the average over latitudes, the resultant $l-v$ plot reveals an elliptical envelope, as forseen by the original EMR hypotheses.

It would be curious indeed if an energetic explosion at the Galactic center left behind no kinematic signature outside of the single plane defined by the 
EMR. In another examination of the latitudinal structure of the EMR, Sofue (1995b) presents evidence that the EMR is really an expanding molecular shell (EMS). Using the ${ }^{13} \mathrm{CO}$ survey data of Bally et al (1987), Sofue finds that the EMS is an oblate spheroid that is pinched around its equatorial waist because the expansion has suffered more impedance there by the higher density in the midplane of the CMZ. He also concludes that the EMS has gaps in it along radial rays that intersect the most prominent interstellar structures in the CMZ, as would be expected if these structures were ponderous and thick enough to block the passage of the EMS. Although this interpretation of the data is not completely unequivocal, it definitely warrants further investigation.

Which of the two competing hypotheses for the 180-pc molecular ring is more correct is not yet clear. Whereas the kinematic response of gas to the Galactic bar should inevitably produce a signature in the $(l, b, v)$ cube looking roughly like the data, episodic explosions at the nucleus might also be both in evidence and inevitable (cf Section 7). Thus, although it appears that the dominant characteristics of the gas kinematics are naturally determined by the bar potential, the final analysis of Galactic center gas dynamics may well require an occasional injection of radial momentum.

\subsection{The Inward Transport of Gas to the Nucleus}

The mass of CMZ material said to be on $X_{2}$ orbits constitutes $85-90 \%$ of the total molecular mass in the Galactic center arena, so the residence time on these orbits is clearly much longer than in the innermost $X_{1}$ orbit. Nevertheless, this reservoir of gas can only be temporary. Angular momentum loss by orbiting disk gas is inevitable, in the face of the many processes acting near the Galactic center. Clouds on $\mathrm{X}_{2}$ orbits circulate with velocities greater than the pattern speed of the bar (estimated at $19 \mathrm{~km} \mathrm{~s}^{-1} \mathrm{kpc}^{-1}$ by Wada et al 1994); they thereby lose angular momentum to the stellar bar by gravitational torques. These clouds are also subject to tidal friction from stars in the bulge (Stark et al 1991), and the most massive of them are doomed to spiral into the Galactic center on time scales of a few times $10^{8}$ years. The magnetic field is another contributor to angular momentum loss, particularly for the less massive clouds; if clouds continually move through a strong $(\mathrm{mG})$, pervasive vertical magnetic field in the inner $100 \mathrm{pc}$ or so, with a relative velocity equal to a substantial fraction of the cloud's orbital velocity, then the magnetic viscous force would cause angular momentum loss on a time scale of $\sim 10^{8}$ years (Morris 1996).

In addition to 1. bar-induced torques, 2. dynamical friction, and 3. magnetic viscosity, several other angular momentum loss processes contribute to the inexorable inward transport of matter: 4. shocks associated with the $\mathrm{X}_{1}-\mathrm{X}_{2}$ transition; 5. viscous drag in the differentially rotating Galactic disk, including 
that resulting from cloud-cloud collisions (von Linden et al 1993, Biermann et al 1993); and 6. dilution of the gaseous disk's specific angular momentum by stellar mass loss material raining down out of the slowly rotating Galactic bulge (Jenkins \& Binney 1994). At present, assessing the relative importance of these mechanisms is rather difficult, but several of them are individually quite important, so gas brought into this arena is destined to migrate into the center on time scales much smaller than a Hubble time.

The rate of mass flow through the ILR, $\dot{M}_{\text {ILR }}$, can be estimated using the mass of molecular gas in the 180-pc ring, $8 \times 10^{6} M_{\odot}$ (Bania 1977, Sofue 1995a), and, following Gerhard (1992), noting that the gas cannot stay on this innermost (cusped) $\mathrm{X}_{1}$ orbit for more than about one orbital period $\left(2 \times 10^{7} \mathrm{yr}\right)$. Thus, $\dot{M}_{\text {ILR }}=\sim 0.4 M_{\odot} \mathrm{yr}^{-1}$. This estimate, which is larger than that of Gerhard because of the larger mass assumed for the 180-pc ring, is quite uncertain, and we regard a range of $\dot{M}_{\mathrm{ILR}} \approx 0.1-1 M_{\odot} \mathrm{yr}^{-1}$ as more appropriate. This range can be compared to the $0.07 M_{\odot} \mathrm{yr}^{-1}$ estimated by Jenkins \& Binney (1994) to be the rate at which the bulge stars within $2 \mathrm{kpc}$ shed mass. The actual contribution from bulge stars is likely to be larger than this, since this estimate is based on the assumption that the bulge is similar to an elliptical galaxy, whereas many bulge stars are younger than this would imply (Lindqvist et al 1992, Rich 1993, Rieke 1993). Of course, if it is to contribute to the mass budget of the Galactic center gas reservoir, the matter shed by bulge stars does indeed have to migrate down to the Galactic plane without being lifted off by a bulge wind. In addition, some fraction of the material moving inward at the ILR may come from further out in the disk, as a result of some of the same angular momentum loss processes, although the torque exerted by the bar outside the corotating region has the opposite sign and may partially counteract the other loss mechanisms. In any case, there appears to be no fundamental problem with finding enough material to maintain the gas inflow at its present rate.

The ratio of the total gas mass inside the 180-pc molecular ring, 4-9 $\times$ $10^{7} M_{\odot}$, to $\dot{M}_{\text {ILR }}$ provides an estimate of $0.4-1 \times 10^{9} \mathrm{yr}$ for the mean residence time for gas in $\mathrm{X}_{2}$ orbits, if we assume a steady state inflow. This range of time scales is similar to that for dynamical friction to extract angular momentum from massive clouds. The gas moves inward from $\sim 150 \mathrm{pc}$ at a mean rate of $0.2-2 \mathrm{~km} \mathrm{~s}^{-1}$, which is comparable to the estimate of $0.3 \mathrm{~km} \mathrm{~s}^{-1}$ for inward radial motion near the solar circle in spiral structure models (Lacey \& Fall 1985) and to the azimuthally averaged inflow velocities found in the bar models of Athanassoula (1992). This inwardly migrating gas meets one of three fates: star formation (0.3-0.6 $M_{\odot} \mathrm{yr}^{-1}$; Güsten 1989, Section 4), a thermally driven Galactic wind or fountain (0.03-0.1 $M_{\odot} \mathrm{yr}^{-1}$; Section 2.3), and accretion into and through the domain of the much smaller-scale circumnuclear disk (0.03- 
$0.05 M_{\odot} \mathrm{yr}^{-1}$; Section 5). Although none of these routes is negligible, star formation evidently dominates.

In principle, the CMZ could undergo a global gravitational instability, creating a "bar within a bar," leading to yet further angular momentum loss and thus to a greatly enhanced inflow rate, as well as to a starburst (Shlosman et al 1989). However, the mass fraction of the gas inside the ILR, 5-10\%, is apparently at or below the limiting value for such an instability. We therefore presume that the list of angular momentum loss processes given above is complete and that the inward flow of gas on 100-pc scales is relatively steady, although the possibility that the Galactic center has undergone substantial convulsions in the past must be kept in mind.

\subsection{Evidence for an $m=1$ Wave}

In addition to the $m=2$ bar mode, evidence exists for an $m=1$ wave with substantial amplitude near the Galactic center. Such a wave would reveal itself as a displacement of the central stellar cluster from the centroid of the bar, of the bar from the center of mass of the Galaxy, and/or as a global tilt of the inner disk of the Galaxy; it would have implications for the stellar velocity field as well. The pronounced longitudinal asymmetry of molecular line emission both from the Galactic center disk population (cf Section 2.2) and from the molecular parallelogram (Section 3.1, Uchida et al 1994a, Blitz 1994) is a long-standing clue that the gas may be responding to an asymmetry in the Galactic potential. A pure $m=2$ bar would show some displacement from $l=0^{\circ}$ because of projection effects, but the predicted offset is considerably less than that observed. Blitz (1994) finds that more distant gas- $-\mathrm{H}$ I at radii out to $750 \mathrm{pc}$ - shares this rotation center offset from the assumed Galactic center: Sgr $\mathrm{A}^{*}$ and its surrounding stellar cluster.

A second clue for an $m=1$ wave in our Galaxy is the velocity displacement with respect to the Local Standard of Rest (LSR) of 30 or $40 \mathrm{~km} \mathrm{~s}^{-1}$ of the appropriate velocity centroids of gas in the 180-pc molecular ring and the $\mathrm{H} \mathrm{I}$ nuclear disk from the $0 \mathrm{~km} \mathrm{~s}^{-1}$ expected in an axisymmetric galaxy (Blitz 1994). A portion of this offset $\left(15 \mathrm{~km} \mathrm{~s}^{-1}\right)$ can perhaps be attributed to motion of the LSR in response to a large-scale triaxial potential (e.g. Blitz \& Spergel 1991a), but the remainder suggests that the entire central gas layer, and perhaps the bulge as well, are in motion, presumably oscillatory, with respect to the Galactic disk. This is reminiscent of observations of lopsided galaxies, i.e. barred spirals in which one spiral arm is much longer than the other (Baldwin et al 1980). In these galaxies, the velocity profiles near the center are invariably found to be highly anomalous, and attempts to pinpoint the rotation center always find it at a large distance from the center of the bar. Curiously, the gas in the circumnuclear disk, lying within several parsecs of the dynamical center of the Galaxy, shows 
no LSR velocity displacement to within about $20 \mathrm{~km} \mathrm{~s}^{-1}$ (Section 5), so the central stellar core and its immediate environment appear to be kinematically independent of the gas motions on scales of a few hundred parsecs.

Theoretically, $m=1$ asymmetries in galaxies may arise in response to gravitational interaction with a passing companion, wherein the time scale for subsequent relaxation is different for the bulge and disk components. However, interaction with another galaxy is not required. Tagger \& Athanassoula (1990), for example, point out that an $m=1$ mode, or wave, can emerge in a galactic disk via nonlinear coupling to $m=2$ spiral waves. From a different perspective, Miller \& Smith (1992) find in numerical experiments that a test particle initially located at the Galactic center is subject to overstable oscillations about the galaxy's mass centroid. This result would imply that a mass concentration such as a galaxy's central stellar cluster should typically appear to be offset from the galaxy center as defined by nearby isophotes. These authors summarize the literature on off-center and lopsided galaxies that can perhaps be understood in this light.

Another form of an $m=1$ wave at the Galactic center is the pronounced tilt of the plane defined by gas in $\mathrm{X}_{1}$ orbits with respect to the large-scale Galactic plane (Liszt \& Burton 1980; Uchida et al 1996a). A tilt of the presumably triaxial bulge has been suggested (Blitz \& Spergel 1991a, Izumiura et al 1995), but $C O B E$ near-IR maps rule out a large tilt (Dwek et al 1995). The tilt of the gas layer, considered as a coherent, propagating warp, can perhaps be understood as a response to the central triaxial bar, even if the major axis of the bar is not itself tilted with respect to the Galactic plane (Binney 1978, Sparke 1984). Longlived warps can also be fed by coupling to $m=2$ spiral density waves (Masset \& Tagger 1995, 1996). For our Galactic center, it remains to be seen whether the tilt of the gas layer and the longitudinal asymmetries near the Galactic center bear any relationship to each other. The understanding of both of these effects is still in its earliest stages.

\section{STAR FORMATION}

The initial conditions for star formation in the Galactic center environment differ dramatically from those found elsewhere in the Galaxy: The temperature, pressure, velocity dispersion, and estimated magnetic field strength were all much larger in the CMZ than in the Galactic disk, in some cases by several orders of magnitude (cf Section 2). Furthermore, potentially collapsing gas clouds are subject to the unusually strong tidal field near the nucleus, capable of overcoming a cloud's self-gravity for cloud densities $<10^{7} \mathrm{~cm}^{-3}$ $(1.6 \mathrm{pc} / r)^{1.8}$, where $r$ is the galactocentric distance (Güsten \& Downes 1980). Consequently, self-gravity can initiate collapse only in the densest clouds. The 
conditions prevailing within the CMZ thus imply an extremely large Jeans mass $\left(\sim 10^{5} M_{\odot}\right)$ and the inhibition of star formation via slow, quasi-static contraction of cloud cores. Nevertheless, star formation may proceed in this manner in the occasional exceptional cloud. The unusually dense and massive Sgr B2 cloud, located $\sim 100 \mathrm{pc}$ in projection from the nucleus, is furiously forming stars (e.g. Mehringer et al 1993, Gaume et al 1995), albeit very probably with an initial mass function (IMF) quite different from that of the Galactic disk. Even in this cloud, however, the possibility has been raised that the star formation was provoked by a cloud collision (Hasagawa et al 1994).

In spite of all of the impediments to star formation within the CMZ, stars are clearly forming there, at many locations. It seems likely that much of the star formation is induced by events external to the clouds, notably by shocks associated with cloud collisions, supernova remnants, and perhaps violent gas outflows from the nucleus. This mode of star formation is likely to lead to an IMF skewed toward relatively massive stars. The lower mass cutoff may be elevated as well (Morris 1993 and references therein). Also, the enhanced metallicity of gas in the CMZ, which can be up to twice the solar value (Lester et al 1981, 1987; Lacy et al 1989; Wannier 1989; Shields \& Ferland 1994), implies a correspondingly large opacity per gram. This affects the IMF by prolonging protostellar collapse, thus allowing more time for matter to accrete.

The global star formation rate in the $\mathrm{CMZ}, \Phi$, has been estimated at 0.3-0.6 $M_{\odot} \mathrm{yr}^{-1}$ by Güsten (1989), who assumed a relatively normal IMF and used the global production rate of Lyman continuum photons derived from the radio continuum flux (Section 2). This estimate is sensitive to the IMF, however; if massive stars are favored, relative to the Galactic disk, then the global rate of star formation should be correspondingly reduced. Güsten (1989) gives a lower limit of $0.05 M_{\odot} \mathrm{yr}^{-1}$ from the luminosity of the discrete far-IR sources measured by Odenwald \& Fazio (1984), but here again a normal IMF is assumed. A proper accounting of the mass budget of interstellar gas in the Galactic center will clearly require new information on the IMF, as well as an improved census of the sites of star formation.

Attempts to understand the spatial distribution of star formation near the Galactic center, and whether, for example, it reflects large-scale shocks in the $\mathrm{CMZ}$, are still in their earliest stages. Infrared imaging has revealed several new star formation sites (Moneti et al 1992, 1994). Another promising approach has been to survey $\mathrm{H}_{2} \mathrm{O}$ masers in IRAS sources (Taylor et al 1993, Levine 1995). After the masers associated with evolved stars were differentiated from those associated with sites of star formation, about a dozen sites of star formation in the Galactic center have been identified. This ongoing survey should eventually reveal large-scale star formation patterns. 
Main sequence stars have been difficult to detect near the Galactic center because of confusion with the more luminous giants, so the IMF has not yet been directly probed. However, access to the upper main sequence is becoming possible with near-infrared observations. Three spectacular clusters of young stars are now known to be located near the Galactic center, each of which contains a sizeable number of exceptionally luminous $\left(10^{6 \pm 0.5} L_{\odot}\right)$ stars: 1 . the well-known cluster occupying the central parsec of the Galaxy, centered roughly on Sgr A*; 2. the Quintuplet cluster, or AFGL 2004, located near the G0.15-0.05 H II region and the NTFs in the Galactic Center Arc (Nagata et al 1990, Okuda et al 1990, Glass et al 1990, Cotera et al 1995, Figer et al 1995); and 3. G0.121+0.017, or Object 17, located near the thermal arched filaments of the Radio Arc (Figure 6; Cotera et al 1994, 1996; Nagata et al 1995; Serabyn $\&$ Shupe 1996). All of these clusters have a population of massive and luminous emission-line stars, including Wolf-Rayet stars (late-type WN and WC stars, notably WN9/Ofpe and WC9) and blue supergiants (luminous blue variables, B[e] stars). The near-IR emission-line spectra of these stars indicate that they have high-velocity winds with speeds of 500 to $1000 \mathrm{~km} \mathrm{~s}^{-1}$ and that many of these stars are helium rich, consistent with their presumed post-main sequence status.

\subsection{Star Formation in the Central Parsec}

The evidence for recent massive star formation within the central parsec has grown over the years (Rieke \& Lebofsky 1982, Lacy et al 1982, Allen 1987, Rieke \& Rieke 1989, Allen et al 1990) and is now rather widely accepted. The cluster of emission-line stars centered on the core of the central stellar cluster has been the most thoroughly studied, and in many respects it is the most remarkable (Allen 1994; Genzel et al 1994; Blum et al 1995a,b; Eckart et al 1995; Krabbe et al 1995; Libonate et al 1995; Tamblyn et al 1996). The presence of at least two dozen $\mathrm{He} \mathrm{I/H} \mathrm{I} \mathrm{emission-line} \mathrm{stars} \mathrm{and} \mathrm{four} \mathrm{M} \mathrm{or} \mathrm{K} \mathrm{supergiants} \mathrm{ascribed} \mathrm{to} \mathrm{this}$ cluster has been interpreted by Krabbe et al (1995) in terms of a modest burst of star formation between 3 and 7 million years ago. The young stars created in this burst are intermingled with the older population of the central stellar core, of which only the giant stars have so far been observed. The young stars dominate the luminosity of the central parsec, but the total mass of the stars formed in the burst is only $\sim 10^{4} M_{\odot}$, far less than the mass of old stars in the central parsec of the Galaxy's central cluster $\left(\sim 10^{6} M_{\odot}\right)$. The newly formed stars have thus likely not yet equilibrated with their elder brethren. This scenario places the newly formed cluster in a relatively brief, windy phase, which should subside in a few million years. The picture is complicated somewhat by the presence of about 10 medium-luminosity, late-type stars in the central 8 arcsecondsobjects which appear to be intermediate-mass AGB stars, signaling that at least one other star formation event took place there within the past $\sim 10^{8}$ years. 


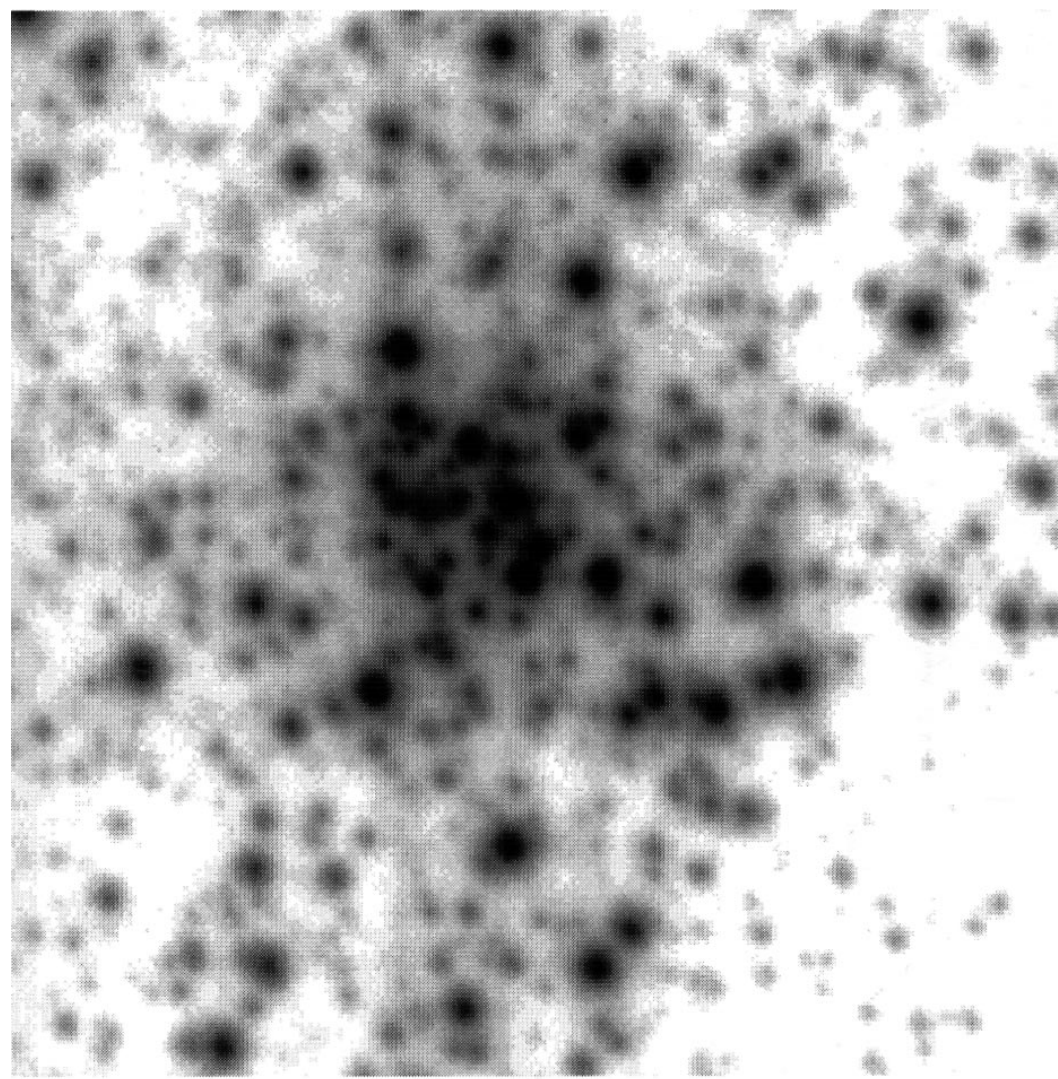

Figure 6 The G0.121+0.017 cluster, imaged at $2.2 \mu \mathrm{m}$ with the NIRC camera on the Keck Telescope (Serabyn \& Shupe 1996).

The implication that the central parsec is the site of repetitive bursts of star formation is perhaps not surprising, given the inevitability of radial inflow of gaseous matter (Section 3), although the factors inhibiting star formation-tidal forces, cloud turbulence, magnetic fields - are probably nowhere more extreme than in the central parsec. The star formation events taking place in the deep gravitational potential well of the Galactic nucleus must be unusually violent events unique to this environment.

\subsection{The Other Major, Young Clusters}

The possibility that the luminous, blue, objects which generate strong winds in the central parsec are something more exotic than young stars (Morris 1993, Eckart et al 1993) was rendered unlikely by the finding of the same kinds of stars 
in two other clusters located well outside the Galaxy's central stellar cluster: the Quintuplet and G0.121+0.017. The luminous post-main sequence stars in these clusters, again identified by their emission-line spectra in the near infrared, constitute a large fraction of the brightest cluster members (Figer 1995, Cotera 1995), especially in G0.121+0.017 (Figure 6), where a dozen stars show $\mathrm{He}$ I and H I emission lines (Cotera et al 1996). If these are WN stars, as Cotera et al suggest, then this one cluster contains $14 \%$ of all known Galactic WN stars. The brightest members of the Quintuplet cluster - the original five-are featureless in spectra measured so far, so their nature is unclear; they may be cocoon-like protostellar objects (Okuda et al 1990) or dust-enshrouded WC9 stars (DF Figer, personal communication). The bright stars dominating the Quintuplet and G0.121+0.017 are likely to be quite massive $\left(50-100 M_{\odot}\right)$; thus, both of these clusters have masses of at least several thousand $M_{\odot}$, and possibly much larger if their IMF is not highly unusual.

The ages of these two remarkable clusters are comparable to that of the young cluster occupying the central parsec. A possible scenario for their formation is that the burst of energy accompanying the formation of stars in the central parsec strongly shocked nearby, massive clouds, inducing a relatively catastrophic gravitational collapse over a region much more widespread than the central parsec. In contrast, the star formation that gave rise to these clusters may resemble what is now going on in Sgr B2, where the star formation is presumably determined by local events. The two known, major, noncentral clusters are likely responsible for ionizing the surfaces of molecular clouds in their immediate environments (Figer 1995, Cotera 1995). However, the cloud near the Quintuplet does not share the velocity of the cluster (Figer 1995); the parent clouds of these clusters may no longer be in evidence.

There are several other sites of star formation near the Galactic center (e.g. Moneti et al 1992, 1994, Lis et al 1994), but no young clusters having the status of the Quintuplet or G0.121+0.017 are known. A survey carried out at $2 \mu \mathrm{m}$ by Figer (1995) over a $60 \times 30$-pc region near the Galactic center revealed no new examples of clusters of emission-line stars, so if there are further instances of such clusters, they are either highly extincted or are located further from the center, in projection, than the known clusters.

One particularly interesting string of H II regions known as G-0.02-0.07, or the Sgr A East H II regions, lies quite close $(10 \mathrm{pc})$ to the Galactic center in projection (Ekers et al 1983, Goss et al 1985). The H II regions, embedded in a ridge within the $50 \mathrm{~km} \mathrm{~s}^{-1}$ cloud (Mezger et al 1989, Ho et al 1991, Serabyn et al 1992b), are neatly aligned adjacent to, and along the edge of, the Sgr A East nonthermal shell source, giving the strong impression that the expandion of the shell has provoked the formation of the stars that ionize the H II regions. 
However, there seems to be a mismatch between the expected expansion time of Sgr A East and the substantially longer time required for stars to form in response to the shock from the expanding shell and then evolve to the main sequence, as these stars have apparently done. Therefore, the origin of this cluster is not yet understood. Furthermore, although the cluster appears to contain at least one evolved emission-line star (Cotera et al 1994), star formation may still be adding to it, as evidenced by the rather high densities inferred for the molecular ridge (Serabyn et al 1992b), where an $\mathrm{H}_{2} \mathrm{O}$ maser is located (Yusef-Zadeh \& Mehringer 1995).

\subsection{The Environmental Effects of Stellar Winds}

The powerful winds emanating from the massive, post-main sequence stars at the Galactic center have a strong effect upon their surroundings, especially when they act collectively, as in the three major clusters described above. The cluster within the central parsec is the only one where the effect of the winds has been demonstrated, and there the result is profound. The 1.5-pc-radius cavity inside the circumnuclear disk (cf Section 5) may be largely swept clear by the cumulative mass outflow winds (Gatley et al 1984, 1986), aside from a few streamers of gas that appear to have enough inertia to maintain their integrity as they move through this region. Consequently, the matter currently accreting onto the central object, Sgr $\mathrm{A}^{*}$, may be almost entirely dominated by the material in the wind (Section 6). Other effects of the wind on gas at small galactocentric radii are described in Section 5.

The most spectacular effect of the Galactic center wind is the ablation of the envelope of the red supergiant, IRS7. The envelope of this well-studied star (e.g. Sellgren et al 1987) is not only ionized by hot stars near the center (Serabyn 1984, Rieke \& Rieke 1989, Yusef-Zadeh et al 1989); it also has an extended, cometary "tail" of ionized gas pointing away from the sources of the wind and showing a pronounced velocity gradient (Yusef-Zadeh \& Morris 1991, Serabyn et al 1991). This apparently is a case of colliding winds: the supergiant has its own radiation-pressure-driven wind (although this modest wind is quickly overcome by the ram pressure of the Galactic wind), and a bow shock is evident on the side of the star facing the wind source (Yusef-Zadeh \& Melia 1992). The hope that the shape and size of this bow shock can allow one to derive the momentum in the wind is diminished by the conclusion of Dyson \& Hartquist (1994) that the ram pressure of the Galactic wind plays no role in determining the size of the large-scale bow shock; the long tail implies that the stellar envelope is clumpy and permeable, so the wind is decelerated primarily in bowshocks around the individual clumps.

The same phenomenon should be happening at some level to the atmospheres of all red giants and supergiants in the central parsec, although no further 
cometary stellar wind tails have yet been observed. Coming years should see more examples of this phenomenon, as sensitive telescopes with improved spatial resolution are brought to bear.

\section{CIRCUMNUCLEAR MATERIAL-DISK OR DEBRIS?}

Our Galaxy's innermost molecular feature is a relatively compact $(<7 \mathrm{pc}$ maximum radius) and dense disk- or torus-like structure that orbits about the center (Genzel et al 1994 and references therein). Although normally referred to as the circumnuclear disk, this nomenclature may actually hide a multitude of sins (Section 5.2). The molecular medium in general extends only to within about $1.5 \mathrm{pc}$ of the center; its inner edge presents a rather sharply defined ionized boundary layer basking in the radiation from the encircled stellar cluster. Much of the filamentary Sgr A West H II region (Figure 7; Killeen \& Lo 1989, Lacy et al 1991, Roberts \& Goss 1993, Yusef-Zadeh \& Wardle 1993), located in the innermost $1.5 \mathrm{pc}$ radius region, can then be attributed to photoionization of the molecular medium's inward-facing surfaces. In order for centrally originating photons to propagate out to the CND's inner boundary, the wind-evacuated central cavity must be comparatively transparent (Becklin et al 1982). However, gas kinematics indicate that several ionized filaments and clumps are found in closer proximity to the center (Serabyn \& Lacy 1985, Serabyn et al 1988, Serabyn 1989, Lacy et al 1991, Herbst et al 1993b, Lacy 1994), as is neutral and molecular gas (Davidson et al 1992, Marr et al 1992, Jackson et al 1993, Pauls et al 1993, Yusef-Zadeh et al 1993, Zhao et al 1995, Telesco et al 1996). Both are likely to be the result of dense gas plunging into the central cavity from the CND or beyond. However, the location of some of the molecular material remains controversial (Liszt \& Burton 1993, Marshall \& Lasenby 1994b). In this section, we discuss recent developments bearing upon this general scenario and also address evidence regarding its shortcomings. As this is a well-studied topic, we limit ourselves to a quick summary.

\subsection{The Standard Model}

The established picture of a clumpy, centrally illuminated torus or disk of largely molecular gas provides a good first-order description of the excitation, distribution, and kinematics of the gas making up the CND. Although a one-toone identification of ionized structures with individual molecular clumps is far from complete, a large and increasing fraction of the ionized gas in the central few parsecs can indeed be accounted for in this manner (Telesco et al 1996). Thus, the intricate distribution of ionized gas evident in the $\mathrm{Ne}^{+} 12.8-\mu$ m image of Sgr A West in Figure 7 (Lacy et al 1991) can likely be attributed largely to photoillumination of the clumpy molecular medium surrounding the center by 


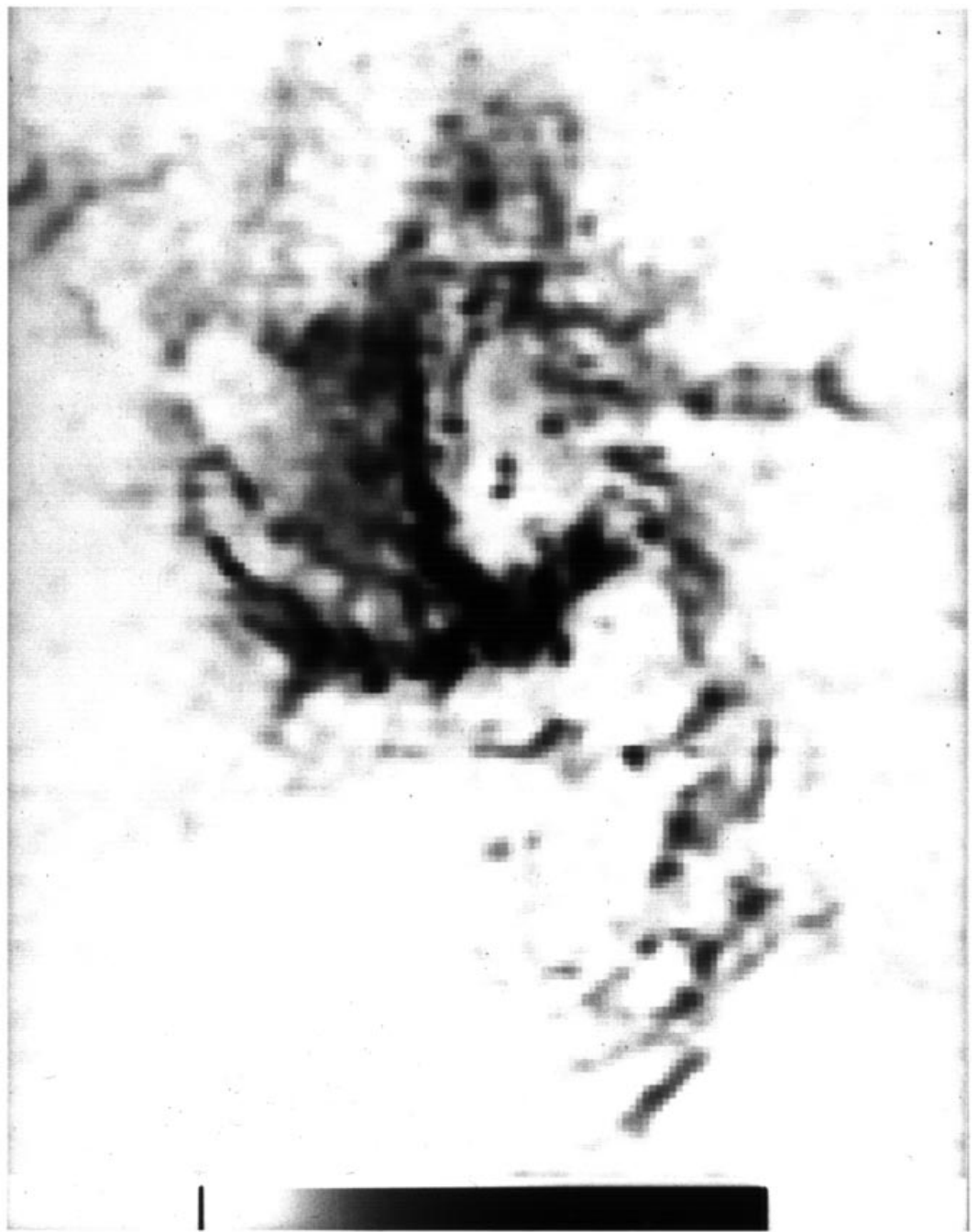

Figure $7 \quad 12.8-\mu \mathrm{m} \mathrm{Ne}^{+}$line emission from the Sgr A West H II region (Lacy et al 1991). The nomenclature for the ionized filaments is as follows: The Western Arc is the diffuse, slightly curved filament crossing the frame from top to bottom to the right (west) of center; the Northern and Eastern Arms are the well-defined filaments extending from the vicinity of the center of the image to the north and east, respectively; and the Bar is the bright, clumpy east-west structure near the center of the image. 
stars in the central cluster. Local heating by individual stars is also observed (Smith et al 1990, Gezari 1992).

Copious measurements have by now probed the molecular medium lurking behind the outermost filaments in Figure 7 (most recently, by Sutton et al 1990, Jackson et al 1993, Marr et al 1993, Marshall \& Lasenby 1994a, and Serabyn et al 1994). The HCN 1-0 map of the inner part of the CND, along with the $90-\mu \mathrm{m}$ dust continuum emission, is shown in Figure 8 . The gas near the CND's inner rim is both hot (a few hundred $\mathrm{K}$ ) and dense $\left(10^{4}-10^{7} \mathrm{~cm}^{-3}\right)$, with line emission consistent with a dense photodissociation region (Genzel et al 1994). The column abundances and excitation states of the ionized and neutral gas components, as well as of the dust component, provide constraints on the incident radiation field's effective temperature and total luminosity (Section 6). The molecular medium is extremely clumpy (with clumps most likely taking the form of tidally sheared streamers), with low filling factor, thereby allowing for penetration of radiation well beyond the CND's innermost edge. Large linewidths ( $~ 40 \mathrm{~km} \mathrm{~s}^{-1}$ ) at the CND's inner edge (Marshall \& Lasenby 1994a) likely reflect a large interclump velocity dispersion, the magnitude of which suggests frequent interclump collisions and shocks. Toward the outer edge of the CND, the linewidths decrease (Serabyn et al 1994), probably due to decreasing clump overlap.

Circular rotation provides a good first-order fit to extant kinematic data on the CND. Most kinematic models yield low values for radially directed velocities, although other interpretations remain possible. The differences in interpretation appear to stem largely from the method of selection of a disk major axis orientation: A major-axis selected to coincide with maximal velocities necessarily yields low radial velocities ( $\lesssim 20 \mathrm{~km} \mathrm{~s}^{-1}$; Jackson et al 1993, Marshall \& Lasenby 1994a, Serabyn et al 1994), while an orientation based on the morphology of the intensity distribution (or any other criterion) can yield a larger radial velocity component (up to $50 \mathrm{~km} \mathrm{~s}^{-1}$; Gatley et al 1986, Gatley \& Merrill 1994; see Fig. 8). A common major-axis orientation may not apply at all radii (or for all molecular species), but even so, speculations about disk warp are likely premature. The most general approach would fit all disk parameters, but few data sets have been complete enough (beyond the CND's inner rim) to warrant such a treatment.

At smaller radii, gas kinematic modelling of the dense, ionized gas filaments in the Sgr A West HII region is possible. The ionized gas shows velocities as high as $\pm 300 \mathrm{~km} \mathrm{~s}^{-1}$, with pronounced velocity gradients in the central 10 arcsec. For several of the inner filaments, eccentric orbits in the field of a point mass reproduce the gas velocities well (Serabyn and Lacy 1985, Serabyn et al 1988, Serabyn 1989, Herbst et al 1993a), under the assumption of gas flow 


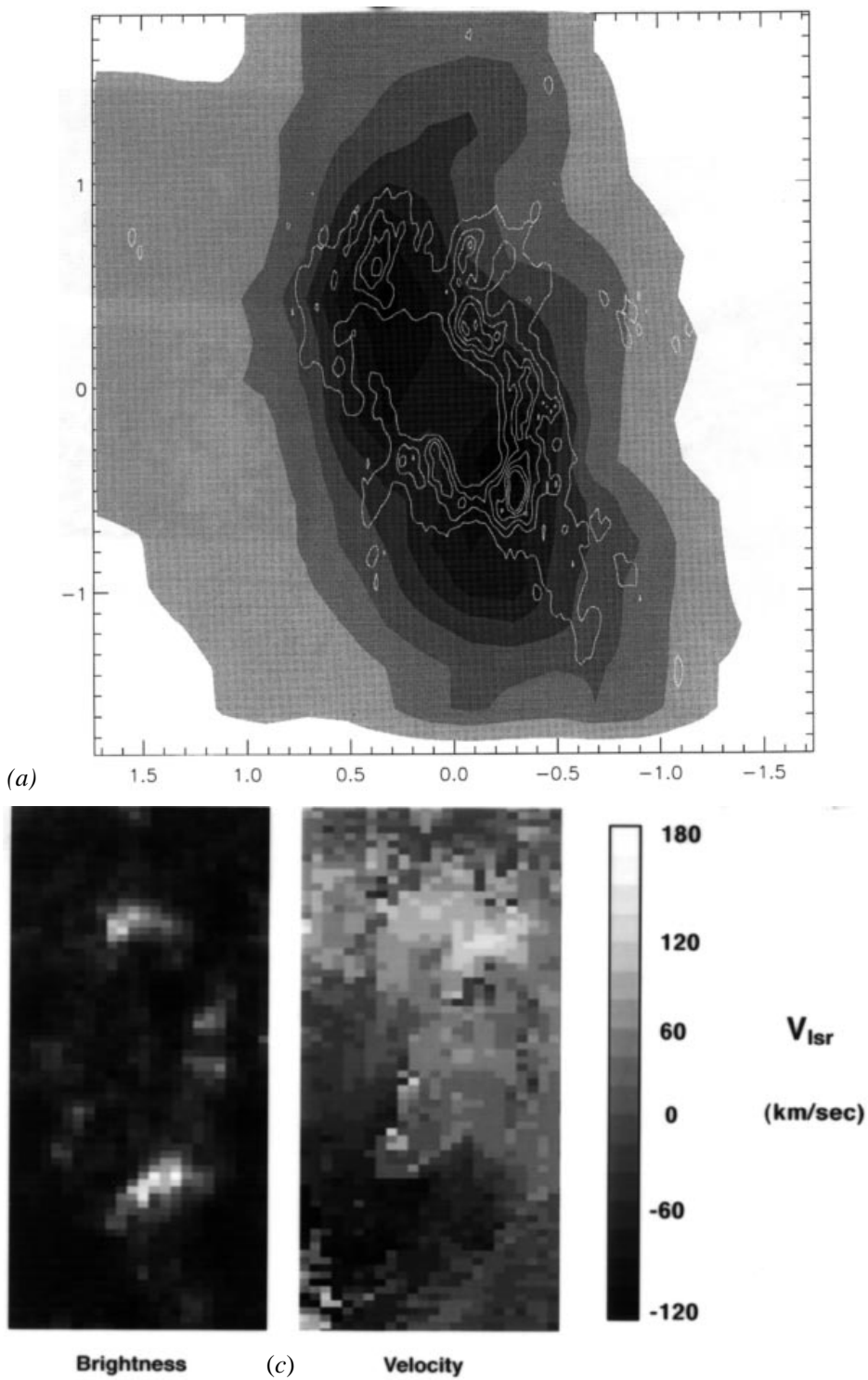

Figure 8 The circumnuclear disk, viewed in the emission of three probes: (a) $90-\mu$ m continuum emission (Davidson et al 1992), and HCN $J=1-0$ emission (Wright et al 1987), (b) 2- $\mu \mathrm{m}$ molecular hydrogen emission (Gatley \& Merrill 1995; I Gatley, personal communication). Panel $c$ shows the velocity field of the $\mathrm{H}_{2}$ emission, indicating that the maximum velocities do not lie at the ends of the apparent major axis of the elliptical emission trace and that the nodes in the velocity distribution do not lie along the apparent minor axis. 
along the filaments. However, flow primarily along the filaments need not be taking place. The alternatives include tidally stretched cloud models (Quinn and Sussman 1985, Lacy et al 1991, Zhao et al 1995), which call for similarly elongated orbits with large radial motions, and the contrasting mini-spiral model of Lacy et al (1991), which allows an interpretation of the data (along a unified Western Arc-Northern Arm feature; see Fig. 7 for nomenclature) in terms of circular orbits. However, growing evidence indicates distinct natures for the Western Arc and the Northern Arm, the former being the inner photoionized boundary of the CND in near circular rotation, and the latter the inner photoionized boundary of a neutral gas feature presumably falling towards the center (the northern intruder; Davidson et al 1992, Jackson et al 1993, Telesco et al 1996). As even the eastern arm and a portion of the innermost bar feature (Fig. 7) have velocity patterns consistent with orbital trajectories transiting the central $0.5 \mathrm{pc}$ (Serabyn 1989), it is difficult to avoid the conclusion that the kinematics of the innermost filaments call for an infall component.

However, while large radial motions are indicated for the ionized filaments interior to the CND (and the underlying molecular material, such as the northern intruder), the CND exhibits relatively small radial motions in most models. Thus, net mass inflow from the CND is probably not the result of small-scale viscosity in a homogeneous disk, but is perhaps more accurately described either in terms of sporadic infall of individual clumps resulting from dissipative inter-clump collisions, or as tongues of gas splitting off the inside edge of the CND as a result of an instability at this interface. Recent estimates for the inflow rate for the CND gas and the material in the central cavity are $\sim 3-5$ $\times 10^{-2} M_{\odot} \mathrm{yr}^{-1}$ (Güsten et al 1987, Jackson et al 1993).

The inner rim of the CND also exhibits emission from vibrationally excited $\mathrm{H}_{2}$, which may result from a strong shock caused by the impinging central wind (Gatley et al 1984, 1986; Fig. 8), although UV fluorescence has not been completely excluded (Pak et al 1996). This wind, arising from stars in the central stellar cluster $(\$ 4)$, probably helps to explain the abrupt density discontinuity at the CND's inner edge, where a quasi-steady equilibrium may be established between the pressures of the wind and the turbulent disk. The impact of the wind upon the disk may also contribute to the large linewidths at the disk's inner edge, and it has been held responsible for creating the radio continuum "streamers" that originate at the interface (Yusef-Zadeh \& Morris 1987a, Yusef-Zadeh et al 1995). However, we note that the sharp inner edge of the CND might also be the result of a discontinuity in the radial gradient of the Galactic mass distribution at that point (Duschl 1988), a density wave in the field of a central point mass (Lacy et al 1991), a hydromagnetic instability (Fridman et al 1994), or as we discuss in the next section, the possible short- 
lived nature of the CND. The wind also appears to affect the distribution of the ionized filaments in the central-most few arcseconds, causing compressed and possibly rippled surfaces on the infalling gas streams (Yusef-Zadeh \& Wardle 1993), and it may be responsible for the formation of the mini-cavity in the gas distribution within the bar (Morris \& Yusef-Zadeh 1987, Yusef-Zadeh et al 1989, 1990, Wardle \& Yusef-Zadeh 1992, Eckart et al 1992, Lutz et al 1993, 1994, Yusef-Zadeh 1994).

\subsection{A Symmetric Disk?}

The major objection to the simple model of the CND described above is that the material beyond the CND's inner edge is distributed highly asymmetrically about the center (Serabyn et al 1986, 1996b; Fukui \& Churchwell 1987, Sutton et al 1990, Marshall \& Lasenby 1994a): at negative Galactic longitudes the disk extends up to $7 \mathrm{pc}$ from the center, whereas at positive longitudes the disk's radial extent is much smaller, $\lesssim 3 \mathrm{pc}$. In galactic latitude, it is the ionized gas which is asymmetrically distributed, with a bright ionized filament (the Western Arc) outlining the positive latitude side of the CND (Fig. 7), but only a weak counterpart in the southeastern quadrant. Although this may result from selective shielding by interior features, the molecular gas distribution along the CND's inner rim is also not entirely symmetric (Jackson et al 1993, Marr et al 1993). In addition, molecular gas extends asymmetrically into the CND's central cavity from the northeast. Kinematically, the velocities are also not quite azimuthally symmetric, although it is not yet clear whether this is the result of non-circular streamlines or merely clumpiness (i.e., regions of "missing" gas). Finally, largely ignored and still unaccounted for is the positional coincidence between the southwestern rim of the nonthermal Sgr A East radio shell source and the thermal Western Arc filament in the Sgr A West HII region (Ekers et al 1983, Yusef-Zadeh \& Morris 1987a, Pedlar et al 1989).

Symmetric models thus probably oversimplify matters. As symmetry implies longevity, asymmetry suggests time dependence, i.e. a short-lived disk. The asymmetric distribution of the CND beyond its inner rim suggests that the current appearance of the CND may result either from the gravitational capture and tidal disruption of a passing molecular cloud or from an energetic disruption of a stable disk. In either case, the relative symmetry of the CND's inner rim is a natural result of the shorter rotational time scales there.

The origin of either of these alternatives may lie in the expanding Sgr A East shell: The atomic carbon map presented in Figure 9 shows a very high degree of anticorrelation between the southern lobe of the CND and the ring of neutral carbon that evidently encloses the Sgr A East radio shell (Serabyn et al 1996b) the carbon ring fades in intensity precisely at the location of the southern lobe of the CND. Thus, either the expansion of the Sgr A East shell has compressed 


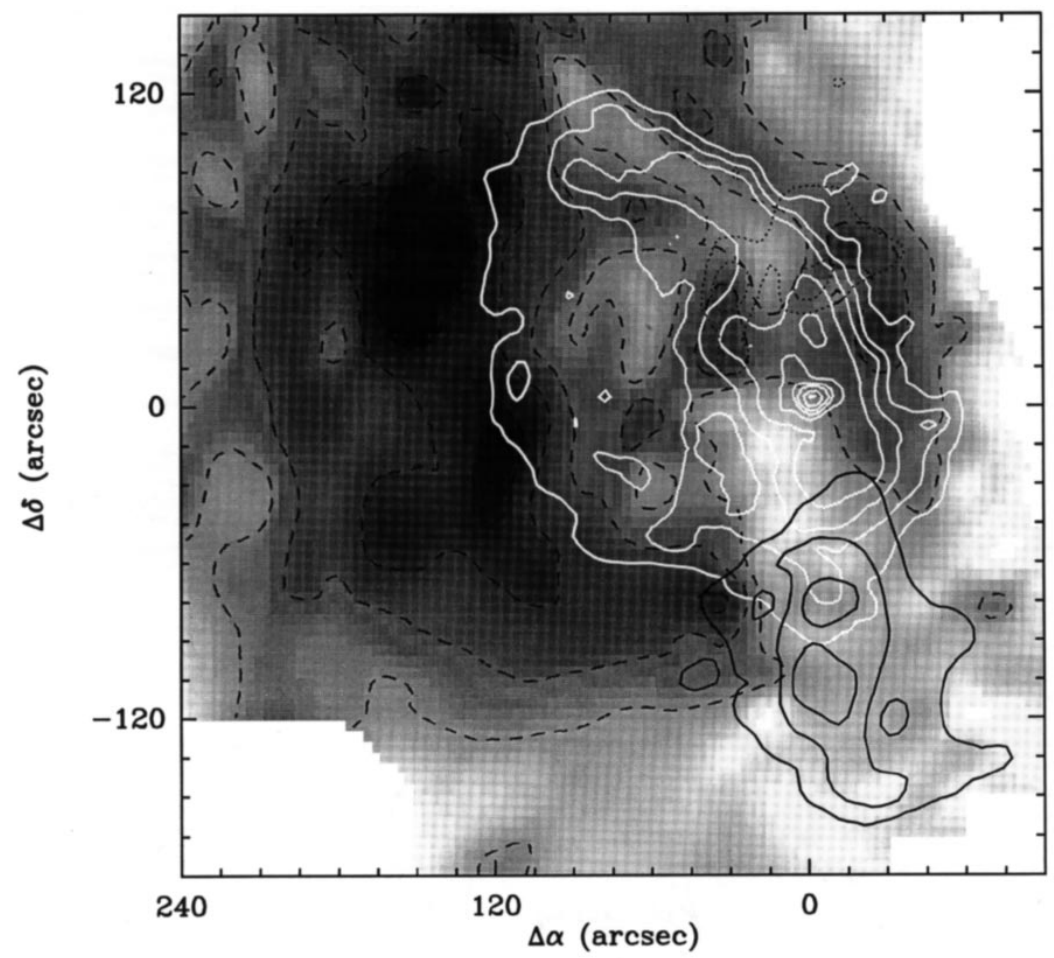

Figure 9 The Sgr A complex, showing the Sgr A East nonthermal radio shell source (outermost white contours), the Sgr A West thermal H II region (fourth white contour and above), the compressed $50 \mathrm{~km} \mathrm{~s}^{-1}$ molecular cloud layers surrounding the expanding radio shell (gray scale and dashed contours), and the circumnuclear disk (solid black and dotted contours). The $(0,0)$ position marks the location of Sgr A*. All maps except the radio continuum map derive from observations of the 492-GHz fine-structure transition of CI (Serabyn et al 1996b). The velocity intervals are$50 \mathrm{~km} \mathrm{~s}^{-1}$ cloud: 30 to $80 \mathrm{~km} \mathrm{~s}^{-1}$; blueshifted CND gas (solid black contours): -130 to $-70 \mathrm{~km}$ $\mathrm{s}^{-1}$; redshifted CND gas (dotted contours): 80 to $130 \mathrm{~km} \mathrm{~s}^{-1}$.

and swept up molecular gas, pushing it toward the center (Mezger et al 1989), where it is now settling into a disklike configuration (in which case the notion of a wind-evacuated central cavity may be unnecessary), or the expansion has disrupted a preexisting and originally more symmetric disk. Alternatively, the geometric relationship could be radiatively highlighted; i.e. the central cluster could be illuminating the inner edge of the Sgr A East shell except where it is shielded by the nearer CND material, but even in this case the expansion of the radio shell must be considered in the context of mechanical interaction with the CND. Although the most likely scenario is not manifest at this point (except that 
Sgr A East is largely behind Sgr A West; Yusef-Zadeh \& Morris 1987a, Pedlar et al 1989), what does emerge clearly is that the expanding Sgr A East shell, and more specifically the molecular material that the shell is compressing (Mezger et al 1989; Genzel et al 1990; Zylka et al 1990; Ho et al 1991; Poglitsch et al 1991; Serabyn et al 1992b, 1996b; Ho 1994), is indeed intimately associated with the CND. As a result of this interaction, the long-lived nature of the CND must be seriously questioned.

\section{THE COMPACT CENTRAL OBJECT}

In common with several categories of external galaxies (Owen et al 1980), our own Galaxy harbors a compact radio source at its center (Lo 1989, 1994; Backer 1994, 1996). Referred to as Sgr A* to differentiate it from the extended Sgr A complex in which it is ensconced, our Galaxy's central radio source shares a unique set of characteristics with its siblings: central location, compact size, high brightness temperature, and a relatively flat radio spectrum. However, its luminosity falls near the low end of the observed range for compact nuclear radio sources, almost certainly the result of selection, as proximity makes our own nuclear source comparatively easy to detect. Thus, the standard argument calling for a massive central black hole on the basis of a prodigious energy output from a small volume is inapplicable in the case of our own Galactic nucleus. Indeed, independent of wavelength, the flux from the vicinity of $\mathrm{Sgr}^{*}$ is quite low compared both to observed active galactic nuclei and to theoretical expectations for the radiative output from an accreting compact object.

\subsection{Source Characteristics}

Although Sgr A* is frequently referred to as a point source, VLBI observations at centimeter wavelengths routinely resolve its emission, showing an apparent source size very closely proportional to $\lambda^{2}$, for wavelength $\lambda$. An axial size ratio of 0.5 is typically observed (Alberdi et al 1993, Lo et al 1993), with a major-axis position angle roughly east-west, and compilations of existing measurements yield a major-axis source size in microarcseconds of $\theta_{\mu \text { as }}=14 \lambda_{\mathrm{mm}}^{2}$ (Jauncey et al 1989, Marcaide et al 1992, Backer 1994, Yusef-Zadeh et al 1994). This behavior is consistent with anisotropic scattering by the intervening interstellar medium, and because nearby maser sources show similar characteristics, the highly magnetized medium in the central few hundred parsecs is a prime candidate for the scattering agent (Frail et al 1994, Yusef-Zadeh et al 1994). Unfortunately, the resultant image broadening prevents direct observation of source structure in $\mathrm{Sgr}^{*}$ at $\mathrm{cm}$ wavelengths (and also masks any possible intrinsic size variation with $\lambda$ ). 
VLBI observations have recently been extended to millimeter wavelengths, yielding tantalizing suggestions of intrinsic source structure (Krichbaum et al 1993, 1994) but as yet no confirmed evidence for a source size exceeding that set by interstellar scattering (Backer et al 1993, Rogers et al 1994). The most stringent observational limit gives an intrinsic source diameter $<130 \mu$ as, or $1.1 \mathrm{AU}$, at $\lambda=3.5 \mathrm{~mm}$ (Rogers et al 1994) and an intrinsic brightness temperature $>1.4 \times 10^{10} \mathrm{~K}$, implicating synchrotron emission. Since the inverse Compton limit and the absence of short-time-scale scintillations at nearmillimeter wavelengths both imply a source diameter $\gtrsim 10 \mu \mathrm{as}$, or $0.1 \mathrm{AU}$ (Kellermann \& Pauliny-Toth 1981, Gwinn et al 1991, Serabyn et al 1992a, Zylka et al 1992), the intrinsic source size at near-millimeter wavelengths is evidently constrained to within an order of magnitude. This scale size is quite interesting from a theoretical perspective. The smallest stable circular orbit about a massive black hole (with a diameter of 6 Schwarzschild radii, or $0.12 M_{6}$ $\mathrm{AU}$, where $M_{6}$ is the black hole mass in units of $10^{6} M_{\odot}$ ) falls near the lower end of the range, for masses suggested by kinematic data $\left(M_{6} \sim 2-3\right.$; Section 6.3). VLBI observations are thus beginning to probe a very telling regime, although it is likely that the shortest millimeter wavelengths, and perhaps even submillimeter wavelengths, will be needed to overcome the scattering handicap and reveal the intrinsic source structure.

The spectrum of Sgr A* is also key to understanding its nature, and the radio source has now been detected at frequencies up to $670 \mathrm{GHz}$ (Zylka et al 1992, 1995; Dent et al 1993). Most observations at frequencies below 100 $\mathrm{GHz}$ show a slowly rising approximately power-law spectrum (Lo 1989), but as moderate variability is established at $\mathrm{cm}$ and $\mathrm{mm}$ wavelengths (Zhao et al 1989, 1992), care is required in deriving the spectral slope. In particular, because Sgr A* shows short-time-scale ( $\$$ month-long), weak ( $\sim$ a factor of 2 ) outbursts at $\mathrm{cm}$ and $\mathrm{mm}$ wavelengths several times per year, with concommitant spectral changes (Wright \& Backer 1993), a concatenation of numerous flux density measurements taken over the years could be misleading. To avoid these concerns, we instead show in Figure 10 nearly simultaneous measurements of the radio/millimeter spectrum of Sgr A* taken inside a span of 12 days in March 1993 with the VLA, OVRO, and CSO-JCMT interferometers (Serabyn et al 1996a). These simultaneous data cover nearly two decades in frequency, from 5 to $354 \mathrm{GHz}$, and the measurements at frequencies below $100 \mathrm{GHz}$ are best fit by a $v^{0.25}$ power law (Figure 10), consistent with earlier spectra. At frequencies above $200 \mathrm{GHz}$, an excess of 1-2 Jy above the extrapolated power law (up to a factor of 2) is evident, and the higher frequency simultaneous data points are in good agreement with prior interferometric and bolometric measurements (Zylka \& Mezger 1988; Zylka et al 1992, 1995; Serabyn et al 1992a; Dent et al 1993). 


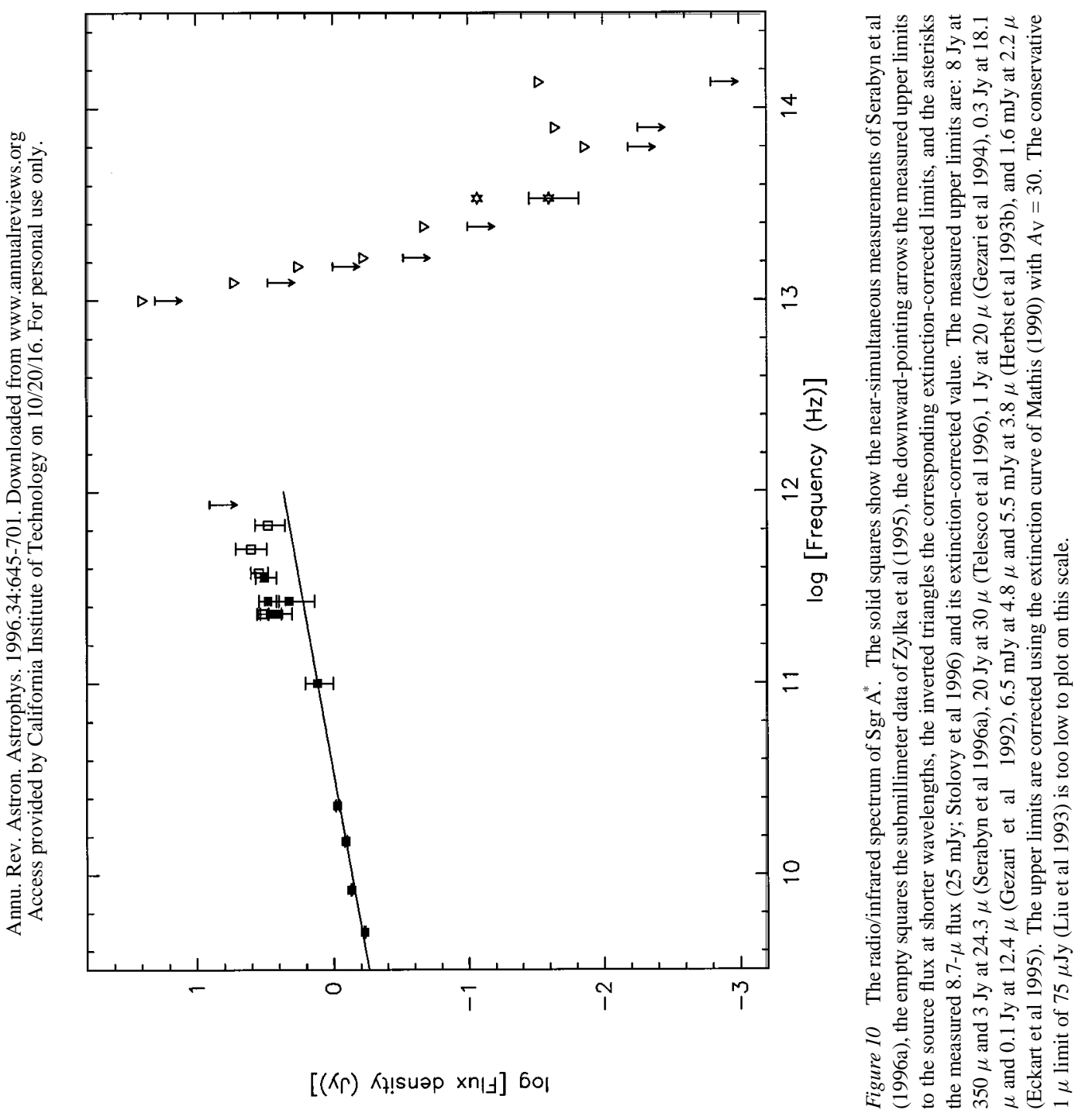


At frequencies beyond those measured interferometrically, the shape of the spectrum remains unsettled. Single-dish measurements between 300 and 670 $\mathrm{GHz}$ yield flux densities of roughly 3-4 Jy for Sgr A* across this range, although an initial report of a $1.5 \mathrm{Jy}$ upper limit at $670 \mathrm{GHz}$ (Dent et al 1993) conflicts. Large error bars leave spectral slopes indeterminate at present, and the extant database is insufficient to definitively address the question of source variability at submillimeter wavelengths. Nonetheless, the fact that Sgr A*'s flux density does not exceed the $4 \mathrm{Jy}$ level even for frequencies approaching $700 \mathrm{GHz}$ suggests that the meager near-millimeter excess is due either to a slight rise in an undulating spectrum, as is seen in external nuclear radio sources at lower frequencies (Owen et al 1980), or perhaps to a final hump just before turnover (Falcke 1996).

Because of its positive spectral index at radio frequencies, the luminosity of Sgr $\mathrm{A}^{*}$ is dominated by its highest frequency emission, making submillimeter and shorter wavelength observations critical for a determination of this quantity. The power-law spectrum to $100 \mathrm{GHz}$ has a total luminosity of $\approx 10^{34}$ $\mathrm{erg} \mathrm{s}^{-1}$, and inclusion of the emission to $700 \mathrm{GHz}$ increases the total luminosity by roughly an order of magnitude, to $1.4 \times 10^{35} \mathrm{erg} \mathrm{s}^{-1}$. At just under $40 L_{\odot}$, this is well below the Eddington limit of even a solar mass object $\left(3.4 \times 10^{4} L_{\odot}\right)$.

Searches have also been carried out for counterparts to Sgr A* at many shorter wavelengths, with success only recently becoming apparent. Several far- and mid-infrared upper limits (Figure 10), as well as the mid-IR detection of a source toward Sgr A* (Stolovy et al 1996), constrain the high-frequency rolloff of Sgr A*'s synchrotron emission. It is clear from the extinction-corrected data at wavelengths shortward of $20 \mu \mathrm{m}$ (Figure 10) that the synchrotron spectrum must cut off between 1 and $10 \mathrm{THz}$, with a falloff at least as steep as $v^{-1}$. The maximum synchrotron luminosity then cannot exceed several hundred $L_{\odot}$. We expect that measurements of the submillimeter and mid-IR spectra will soon be dramatically improved by the new generation of high-resolution cameras.

In the near infrared, where angular resolution and sensitivity have increased markedly in the past decade, no definitive counterpart to Sgr $\mathrm{A}^{*}$ has yet been identified, owing primarily to confusion by surrounding stellar sources. Increased pointing accuracy has eliminated several candidate detections, and most recently, Eckart et al (1995) have found a compact cluster of half a dozen nearIR sources located within a few tenths of an arcsecond of the position of Sgr $\mathrm{A}^{*}$ (Figure 11), possibly representing a central cusp in the stellar distribution. While intriguing in its own right, this result makes the identification of any nonstellar near-IR source that may be associated with Sgr A* itself problematic. At the same time, the extremely low limits $\left(\mathrm{m}_{K} \gtrsim 14\right.$, Figure 10$)$ to the 


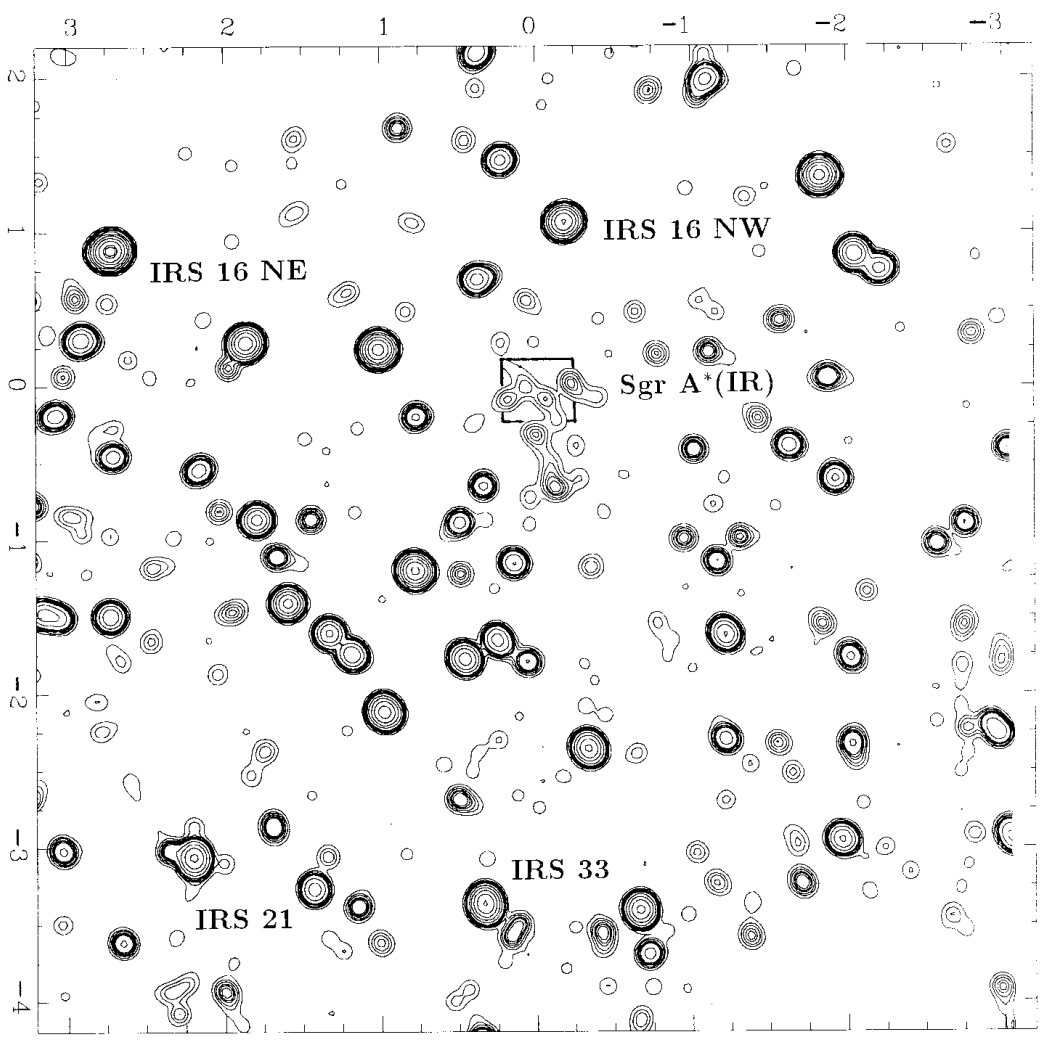

Figure 11 High-resolution K-band image of the vicinity of Sgr A*, from Eckart et al (1995).

possible near-IR flux attributable to Sgr A* severely constrain accretion disk models (Section 6.2).

At visual and UV wavelengths, interstellar extinction obscures the Galactic center completely, but constraints on the radiation field at these wavelengths can nevertheless be derived from the excitation of the ISM in and surrounding the central parsec (Becklin et al 1982, Lacy et al 1982, Serabyn et al 1985, Davidson et al 1992, Maloney et al 1992, Genzel et al 1994, Shields \& Ferland 1994, Zylka et al 1995, Telesco et al 1996). The result for a single-component cloud model is a radiation temperature of about $35,000 \mathrm{~K}$, an ionizing photon flux of $2 \times 10^{50} \mathrm{~s}^{-1}$, and a total luminosity of $\approx 2 \times 10^{7} L_{\odot}$. However, because stellar sources apparently dominate the production of Lyman continuum photons in the central parsec (Rieke et al 1989; Krabbe et al 1991, 1994; Tamblyn \& Rieke 1993), the optical/UV flux in the central parsec arising from Sgr A* itself is 
likely only a small fraction of the total $\left(\lessgtr 10^{6} L_{\odot}\right)$ and is hence difficult to determine reliably.

At an energy of roughly $1 \mathrm{keV}$, it again becomes possible to search for emission from the direction of Sgr A*. A 5-arcmin resolution GRANAT ART-P map at 4-20 keV shows emission from the general direction of the extended Sgr A source (Syunyaev et al 1991, Pavlinsky et al 1994), but high- resolution 2-10 $\mathrm{keV}$ ASCA data (Koyama et al 1996) show the main emission to be associated not with Sgr A* but with the Sgr A East shell source. The $2-10 \mathrm{keV}$ flux from Sgr A* itself is then limited by the ASCA observations to $\sim 10^{35} \mathrm{erg} \mathrm{s}^{-1}$ (Koyama et al 1996), which is an order of magnitude below the large-beam GRANAT estimate and more consistent with the $1.5 \times 10^{35} \mathrm{erg} \mathrm{s}^{-1}$ seen below 4 $\mathrm{keV}$ by Watson et al (1981). The lower ASCA flux is also more consistent with data in the softer 1.2-2.5 keV ROSAT band (Predehl \& Trümper 1994), where a source coincident with Sgr A* was also found. Comparison of the ROSAT and GRANAT data had earlier suggested the need for an anomalously high extinction at long X-ray wavelengths, but this need is removed by the lower ASCA flux actually attributable to the compact source. Of course, source variability may affect this discussion. At higher energies (35-150 keV), no source was detected toward Sgr A* by the Sigma/GRANAT telescope (Goldwurm et al 1994). Thus, the total X-ray flux that arises in a small enough region to be attributable to Sgr $\mathrm{A}^{*}$ itself is no more than a meager $2.5 \times 10^{35} \mathrm{erg} \mathrm{s}^{-1}$.

\subsection{Source Models}

The preceding section establishes that Sgr A* is a rather weak source across the electromagnetic spectrum, with a luminosity of at most a few hundred $L_{\odot}$ at radio and X-ray wavelengths. The intermediate-frequency situation is not as clear, but allowable near-IR/optical/UV luminosities have decreased markedly over the past decade (by a factor of 100 at $\mathrm{K}$ band), ruling out luminosities for Sgr $\mathrm{A}^{*}$ in excess of $10^{6} L_{\odot}$. Because of the low radio and X-ray luminosities definitively attributable to Sgr $\mathrm{A}^{*}$, accretion onto a stellar source cannot be excluded solely on energetic grounds. However, the radio spectrum of Sgr A* is much flatter than that of a pulsar, too stable for a mass-transfer binary, and much more energetic than typical examples of either (Lo 1989). Furthermore, as proper motion studies (Backer 1996) establish a lower limit to the mass of Sgr $\mathrm{A}^{*}$ of at least $100 M_{\odot}$, and possibly as high as $1000 M_{\odot}$ (depending on whether one assumes kinetic equilibrium with low- or high-mass stars, respectively), stellar sources are effectively ruled out. Thus, models advanced recently to account for the emission from this unique radio source are all variants on the general scenario of accretion onto a massive compact object.

Proposed models differ widely in their assumptions, including the size of the central mass, the accretion rate and geometry, the radiative mechanisms 
and efficiency, the source opacity, and the disposition of infalling material. Central masses of 500-3 $\times 10^{6} M_{\odot}$ appear in different models (Falcke et al 1993; Ozernoy 1993, 1994; Melia 1994a,b; Falcke 1996; Narayan et al 1995), and accretion rates vary even more widely, from $10^{-10}$ to $10^{-4} M_{\odot} \mathrm{yr}^{-1}$, the high end of which is coincidentally the maximum possible average accretion rate over a Hubble time. Emission geometries range from spherical to disk-like to jet-like, with the curious result that the same radio spectrum can apparently be fitted (to some level of accuracy) with either a jet-like outflow or an accretion inflow. The radio spectrum as measured to date thus may be less than definitive, and indeed, models running the gamut from optically thick to thin have been proposed (de Bruyn 1976, Reynolds \& McKee 1980, Duschl \& Lesch 1994). Higher frequency continuum flux measurements and VLBI imaging will no doubt aid in discriminating between models, since a definitive measure of source geometry, size, and cutoff frequency would provide tangible constraints.

Potentially one of the most telling probes of SgrA* may well be its spectrum beyond the radio (i.e. synchrotron) regime. It is too soon to tell whether mid-IR emission might arise from the tail of the synchrotron spectrum (which is at least as steep as $v^{-1}$ ), a dust component, inverse-Compton scattered photons, or from an accretion disk photosphere. With only upper limits available from the near infrared through the UV, definitive model fitting is unfortunately not yet possible at these wavelengths either. It has, however, long been clear that standard accretion disk photospheres are in conflict with stringent nearIR/UV source constraints (Rieke \& Lebofsky 1982, Lacy et al 1982, Rieke et al 1989). The only potential means of evading the near-IR constraints, for a classical thin disk, is to assume a disk orientation very close to edge-on, certainly a distinct possibility (we are, after all, located in the plane of the Galaxy), but the UV constraint arising from the excitation of the surrounding medium (after removal of the stellar UV contribution) is relatively independent of disk orientation. Furthermore, general-relativistic light bending tends to isotropize emergent radiation, rendering even an edge-on disk visible (Falcke \& Heinrich 1994, Hollywood \& Melia 1995); hence, disk inclination may not be a panacea after all. Thus, near-IR/UV limits remain extremely restrictive. Finally, high-resolution X-ray data (Watson et al 1981, Koyama et al 1996) also provide very tight luminosity constraints.

How do existing models fare? Four schools of thought have recently emerged in rather distinct corners of parameter space, and we briefly outline the salient points. The first two models are related; both are based on the idea that a massive black hole would capture and accrete a sizable fraction of the observed stellar mass-outflow wind in its vicinity (Ozernoy 1989), a scenario that has the advantage of properly accounting for the specific environment of Sgr A*. 
Expanding on this idea, Ozernoy $(1993,1994)$ and Mastichiadis \& Ozernoy (1994) claim that the observed radio and X-ray luminosities of Sgr A* determine upper limits to its mass of $\sim 500 M_{\odot}$ and $\sim 6000 M_{\odot}$, respectively. Because the Bondi-Hoyle accretion rate is proportional to the square of the central mass, such a low mass results in very slow accretion $\left(10^{-10} M_{\odot} \mathrm{yr}^{-1}\right)$, and so little near-IR radiation is produced. However, the low mass also introduces a small length scale, implying optically thick synchrotron emission at high frequencies, and so a further emission component not currently in the model is needed to account for the flat low-frequency spectrum observed (Falcke 1996). Beyond this, a detailed prediction for the source spectrum would certainly aid in evaluation.

A similar Bondi-Hoyle accretion scenario is considered by Melia (1994a,b), but diametrically opposite conclusions are reached: A central mass of 1-3 $\times 10^{6} M_{\odot}$ is called for, and an extremely high accretion rate $\left(1.6 \times 10^{-4} M_{\odot}\right.$ $\mathrm{yr}^{-1}$ ) emerges, necessitating an edge-on disk unless a general-relativistic treatment is applied (Hollywood \& Melia 1995). However, although the lowfrequency radio spectrum (below $100 \mathrm{GHz}$ ) can be fitted, the turnover frequency predicted tends to be too low, no excess emission at submillimeter wavelengths is predicted, and the most recent near-IR/UV and X-ray constraints to the flux from Sgr A* are likely violated.

Of course, the near-IR and UV luminosity problems are obviated if the accreting material radiates with low efficiency. This can occur if electrons and ions are decoupled in temperature in the inner parts of the disk (Rees et al 1982, Narayan et al 1995), a situation that can arise because the viscously heated heavy ions cannot easily transfer energy to the much lighter electrons via two-body collisions. Thus, the more efficient radiators do not have ready access to the thermal energy, and the energy is advected with the ions' flow (Abramowicz et al 1995, Narayan \& Yi 1995). To remove angular momentum on a time scale short compared to the ion-electron equilibration time scale, a reasonably high viscosity is also needed, implying radial infall rates higher than in a standard thin disk $\left(10^{-7}-10^{-5} M_{\odot} \mathrm{yr}^{-1}\right.$ in Narayan et al 1995). Accreting material is then able to fall through the Schwarzschild radius before radiating away its thermal energy content, yielding a "dim disk" in the nearIR/optical/UV range. The resulting spectrum (Narayan et al 1995) consists of a series of roughly equal energy humps, due to (in increasing frequency) synchrotron, inverse-Compton, and thermal bremsstrahlung emission. A central mass of rougly $10^{6} M_{\odot}$ provides a good match to the submillimeter luminosity, but not to the radio spectrum (without the addition of an ad hoc component), and there may also be difficulties in fitting the X-ray and submillimeter fluxes in a single model, especially when the data of Koyama et al (1995) are considered. However, because plasma instabilities may serve to couple the ions and 
electrons much more effectively than single-particle collisions, this scenario is based on an untested assumption.

Finally, in analogy with external galaxies, a core-jet model has been suggested (Blandford \& Königl 1979, Falcke et al 1993, Falcke \& Heinrich 1994, Falcke 1996), in which the observed radio emission arises not in the accreting material, but in an ensuing jet-like outflow. This model thus has the specific prediction that at high enough angular resolution, a jet should be seen, and it is also the only model that predicts a slight excess of emission at submillimeter wavelengths. The latter results from the emission from the jet nozzle, the smallest scale structure (Falcke 1996). The predicted accretion rate is $\sim 10^{-7} M_{\odot}$, a mid-range value, and is thought to be set by long viscous time scales in a remnant steady-state accretion disk. Because the accretion rate is governed not by the capture rate, but by viscosity, the central mass is not well constrained. Possible drawbacks to this model are the large discrepancy between the Bondi capture rate and the low steady-state accretion rate required, the fact that no jet has yet been conclusively detected, and the lack of a prediction for the X-ray emission.

While several of these models show promise, the contrast between them also serves to highlight the continuing scarcity of definitive source-structure information. However, with continually improving observational capabilities throughout the electromagnetic spectrum, this situation will no doubt soon improve.

\subsection{The Central Mass}

Clearly one of the most vital of Sgr A*'s unknown parameters is its mass, which cannot be determined uniquely from models of the emerging radiation. Nonetheless, mounting observational evidence over the past decade consistently shows that line-of-sight velocities increase toward the center (inside the central $0.5 \mathrm{pc}$ or so), for both the gas and the stars (Lacy et al 1980, 1982, 1991; Serabyn \& Lacy 1985; Serabyn et al 1988; McGinn et al 1989; Sellgren et al 1990; Herbst et al 1993a; Krabbe et al 1995; Haller et al 1996). The conversion of these findings to an enclosed mass distribution is fraught with uncertainties, for both the stellar and gaseous components, but different techniques yield remarkably good agreement both for the size $\left(2-3 \times 10^{6} M_{\odot}\right)$ of the central dark mass concentration (observationally constrained to lie within the central few tenths of a parsec) and for the distributed stellar mass, $\sim 3 \times 10^{6} r_{\mathrm{pc}} M_{\odot}$, just beyond the core radius of the central cluster (cf Rieke \& Rieke 1988, McGinn et al 1989, Lindqvist et al 1992, Serabyn et al 1994, Haller et al 1996). Although the radial size constraint on the dark mass concentration cannot be used to tie the mass excess directly to Sgr $\mathrm{A}^{*}$, new imaging techniques now being employed are pushing observations ever closer to the center, where the 
discrimination will no doubt improve. In particular, the possible stellar cusp around Sgr A* may soon provide important proper motion data (Eckart et al 1995).

\section{THE EPISODIC NATURE OF ACTIVITY AT THE GALACTIC CENTER}

The inexorable inward flow of matter in our Galaxy makes nuclear activity inevitable; if the accretion rate onto a presumed central black hole of mass $M_{6}$ were only $0.02 M_{6}(0.1 / \epsilon) M_{\odot} \mathrm{yr}^{-1}$, where $\epsilon$ is the efficiency with which the accretion energy is radiated away (often estimated at $\sim 0.1$ ), then the nucleus would emit at the Eddington rate, with an energy output of Seyfert proportion, $\gtrsim 10^{43} \mathrm{ergs} \mathrm{s}^{-1}$. Given the mass flow budget outlined in Sections 3.2 and 5.1, a time-averaged mass flow rate into the central parsec equal to this value is plausible. However, the current accretion rate and luminosity of $\mathrm{Sgr} \mathrm{A}^{*}$ are many orders of magnitude smaller than this (Section 6). It therefore appears that the Galactic center may now be in a lull between brief accretion events. The massive young stars clustered within the inner parsec are indicative of a substantial mass accumulation event within the past $10^{7} \mathrm{yr}$, while the probably inward-moving circumnuclear disk likely heralds the succeeding accretion epoch, possibly within the coming $10^{5}-10^{6} \mathrm{yr}$.

The implied limit cycle is one that begins with the relatively sudden $\left(\lesssim 10^{4}\right.$ yr) appearance of gas within the gravitational potential well defined by the core of the central stellar cluster (0.2-1.2 pc; Rieke \& Lebofsky 1987, Allen 1994, Rieke \& Rieke 1994, Eckart et al 1995). This would occur, for example, if the inward radial velocity of the CND were to be as high as $50 \mathrm{~km} \mathrm{~s}^{-1}$ during its last stages of inward migration, or if the CND underwent a strong disk instability during its descent, causing unusually rapid transfer of angular momentum outwards. Alternatively, dissipative intercloud or interclump collisions might be responsible for sending material rapidly inwards. The gas arriving in the inner few tenths of a parsec has no exit other than to accrete onto a compact object (either a supermassive black hole or one of a collection of smaller ones; Morris 1993, Lee 1995) or to form stars. However, as discussed in Section 4 , star formation is inhibited until either the density grows above a few times $10^{7} \mathrm{~cm}^{-3}$ or the gas is subjected to a violent shock. Such a shock may be provided by fast-moving cloud collisions in the small central volume or by the energy emerging from an accretion disk around the supermassive black hole. The combination of accretion energy, in the form of radially propagating winds and radiation, coupled with the energy released in the formation of dozens, if not hundreds, of massive stars, reverses the infall of gas and begins a period 
of winds (initially from the massive $\mathrm{O}$ stars, and later from the post-main sequence WR-type stars), aided considerably by radiation pressure on dust. This would be the moment at which an expanding molecular ring, if such exists in our Galaxy, could be launched. However, gas clouds initially in the near vicinity of the nucleus can only be pushed to a relatively large radius under the most extreme circumstances; for a 0.5 -pc diameter cloud of density $10^{5} n_{5}$ $\mathrm{cm}^{-3}$, for example, the ratio of the outward radiative to the inward gravitational accelerations at galactocentric distance $r_{\mathrm{pc}}$, even assuming a Seyfert-like central luminosity of $10^{43} \mathrm{ergs}$, is only $\sim 0.1 n_{5}^{-1} r_{\mathrm{pc}}^{-1}$. The ratio of the ram pressure acceleration of that same cloud caused by a steady wind carrying $\dot{M} M_{\odot} \mathrm{yr}^{-1}$ at a velocity of $1000 v_{3} \mathrm{~km} \mathrm{~s}^{-1}$ to the gravitational acceleration is $4 \dot{M} v_{3} n_{5}^{-1} r_{\mathrm{pc}}^{-1}$. Thus, an exceptionally large mass outflow rate would have to be sustained for an extended time period $\left(\sim 10^{4}\left(\dot{M} v_{3}\right)^{-1} n_{5} r_{\mathrm{pc}}\right.$ yr $)$ to give a significant radial impulse to even a modest cloud starting near the center. Alternatively, in place of a steady wind, this mass outflow could be accomplished with a succession of at least $10^{2}-10^{3}$ supernovae occurring within the central parsec on a similar time scale. If and when these conditions are met, the radially moving gas would mix with orbiting gas, raising its specific angular momentum and preventing it from simply falling back in on a free-fall time scale.

At this stage, the Galactic nucleus would resemble that of an emission-line galaxy, a starburst galaxy, or even an AGN, depending on how much mass had accumulated before the reversal took place and on whether the central accretion disk generates more energy than the luminous, newborn stars. Also, those stars would resemble the kinds of stellar objects that have been considered as the source of activity in emission-line galaxies [e.g. "warmers" (Terlevich \& Melnick 1985) or the stars in Wolf-Rayet galaxies (Conti 1991)].

As these massive stars evolve on a time scale of a few million years, their population declines as some of them become supernovae, and the total stellar luminosity decreases. The luminosity of the accretion disk also declines, as it exhausts the supply of gas with which it was impulsively endowed during the relatively brief accumulation phase. The surrounding gas transfers its angular momentum outwards by the processes described in Section 3.4 and migrates back in, against the resistance of the winds from the central stars, for as long as those stars stay in a windy stage of evolution. In broad terms, this is the state in which we now apparently find the Galactic center. However, the gas is not smoothly distributed, so the timing of the recurrent accretion episodes and the manner in which they occur can only be characterized stochastically. In a picture proposed by Sanders (1981), activity at the Galactic center is initiated when a cloud passes close enough to be accreted. We adopt that picture here, noting that a cloud that is "captured" near the Galactic nucleus is likely to 
first form a sheared, lumpy, and asymmetric circumnuclear disk much like the observed CND. The task before us is to unravel the final stages of this recurrent process by understanding how rapidly the CND is now moving inward and then how the gas will behave as it descends upon the denizens of the center.

Interstellar gas is not the only possible source of matter for the accretion disk around the presumed supermassive black hole at the center, since stars whose orbits carry them within the central object's Roche limit are susceptible to tidal disruption (Hills 1975, Ozernoy 1979, Lacy et al 1982, Rees 1988, and references therein). Some fraction of the resulting debris settles into a disk that can have a large accretion rate and a consequent luminosity approaching the Eddington luminosity. Phinney (1989) estimates that, in the Galactic center, this happens about once every $10^{4} \mathrm{yr}$, with the accompanying luminosity impulse lasting 10-100 yr. This settling may affect the X-ray appearance of the Galactic center by producing, for example, a large flux that can be scattered in the $6.4-\mathrm{keV}$ iron line (Koyama et al 1996), thereby giving rise to an iron-line glow persisting for hundreds of years after the initial, brief, production event. Another potential consequence of stellar disruption arises directly from the ejecta created during the tidal disruption event. Khokhlov \& Melia (1996) note that half the stellar mass is asymmetrically ejected with an energy well in excess of that of a supernova, and so they propose that the energetic and asymmetric Sgr A East shell source was created in this manner. This stellar snacking will not typically have much effect on the limit cycle in which Galactic center gas is engaged; however, if it is timed to occur within the $\lesssim 10^{4}$-year interval during which gas is descending at a large rate upon the nucleus, then the pulse of radiation (and perhaps matter, in the form of a jet or a wind) can play a pivotal role in inducing star formation within the central parsec and controlling the rate at which gas accumulates onto the central black hole.

Finally, we remark that a profound shift of emphasis has taken place in Galactic Center research since the time of Oort's (1977) review. That review stressed outflows and violent ejections from the Galactic nucleus, whereas we have traced the thread of inflow and accretion. The recognition of the bar-like nature of the central potential has dramatically reduced the need for explosive origin models for observed kinematic structures. Still, winds and jets remain an important part of the story of Galactic center activity (e.g. Sofue 1994), although the ultimate driver for that activity is the far larger amount of mass flowing toward the Galactic center.

\section{ACKNOWLEDGMENTS}

We thank our many colleagues who avidly contributed with discussions, unpublished data, preparation of figures, and/or readings of the preliminary manuscript: 
R Blandford, J Carlstrom, JA Davidson, D Dowell, I Gatley, RH Hildebrand, JH Lacy, DC Lis, N Mastrodemos, S Phinney, DL Shupe, S Stolovy, K Uchida, and $\mathrm{MCH}$ Wright. We also appreciate little Nicholas Serabyn's unrelenting interest in the flavor of the manuscript.

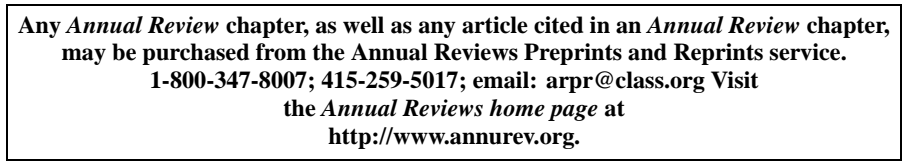

\section{Literature Cited}

Abramowicz MA, Chen X, Kato S, Lasota J-P, Regev O. 1995. Ap. J. Lett. 438:L37-39

Aitken DK, Briggs GP, Roche PF, Bailey JA, Hough JH. 1986. MNRAS 218:363-84

Aitken DK, Smith CH, Gezari D, McCaughrean M, Roche PF. 1991. Ap. J. 380:419-28

Alberdi A, Lara L, Marcaide JM, Elosegui P, Shapiro II, et al. 1993. Astron. Astrophys. 277:L1-4

Allen DA. 1987. See Backer 1987, pp. 1-7

Allen DA. 1994. See Genzel \& Harris 1994, pp. 293-304

Allen DA, Hyland AR, Hillier DJ. 1990. MNRAS 244:706-13

Altenhoff WJ, Downes D, Pauls T, Schraml J. 1978. Astron. Astrophys. Suppl. 35:23-54

Anantharamaiah KR, Pedlar A, Ekers RD, Goss WM. 1991. MNRAS 249:262-81

Armstrong JT, Barrett AH. 1985. Ap. J. Suppl. 57:535-70

Arons J, Max CE. 1975. Ap. J. Lett. 196:L77-81

Athanassoula E. 1992. MNRAS 259:345-64

Backer DC, ed. 1987. AIP Conf. Proc. No. 155, The Galactic Center. New York: AIP. 204 pp.

Backer DC. 1994. See Genzel \& Harris 1994, pp. 403-10

Backer DC. 1996. See Blitz \& Teuben 1996. p. 193

Backer DC, Zensus JA, Kellerman KI, Reid M, Moran JM, Lo KY. 1993. Science 262:141416

Baldwin JE, Lynden-Bell D, Sancisi R. 1980. MNRAS 193:313-19

Bally J, Stark AA, Wilson RW, Henkel C. 1987. Ap. J. Suppl. 65:13-82

Bally J, Stark AA, Wilson RW, Henkel C. 1988. Ap. J. 324:223-47

Bally J, Yusef-Zadeh F, Hollis JM. 1989. See Morris 1989, pp. 189-93

Bania TM. 1977. Ap. J. 216:381-403

Becklin EE, Gatley I, Werner MW. 1982. Ap. J. 258:135-42
Benford G. 1988. Ap. J. 333:735-42

Bennett CL, Fixsen DJ, Hinshaw G, Mather JC, Moseley SH, et al. 1994. Ap. J. 434:587-98

Biermann PL, Duschl WJ, von Linden S. 1993. Astron. Astrophys. 275:153-57

Binney J. 1978. MNRAS 183:779-97

Binney J. 1994. See Genzel \& Harris 1994, pp. 75-86

Binney J, Gerhard OE, Stark AA, Bally J, Uchida KI. 1991. MNRAS 252:210-18

Blandford RD, Königl A. 1979. Ap. J. 232:3448

Blitz L. 1994. In Physics of the Gaseous and Stellar Disks of the Galaxy, ed. I King, pp 1-13. San Francisco: ASP

Blitz L, Teuben PJ ed. 1996. IAU Symp. No. 169, Unsolved Problems of the Milky Way. Dordrecht: Kluwer. In press

Blitz L, Binney J, Lo KY, Bally J, Ho PTP. 1993. Nature 361:417-24

Blitz L, Bloemen JBGM, Hermsen W, Bania TM. 1985. Astron. Astrophys. 143:267-73

Blitz L, Spergel DN. 1991a. Ap. J. 370:205-24

Blitz L, Spergel DN. 1991b. Ap. J. 379:631-38

Bloemen JBGM, Deul ER, Thaddeus P. 1990. Astron. Astrophys. 233:437-55

Bloemen JBGM, Strong AW, Blitz L, Cohen RS, Dame TM, et al. 1986. Astron. Astrophys. 154:25-41

Blum RD. 1995. Ap. J. Lett. 444:L89-92

Blum RD, DePoy DL, Sellgren K. 1995a. Ap. J. 441:603-16

Blum RD, Sellgren K, DePoy DL. 1995b. Ap. J. Lett. 440:L17-20

Bottema R, Sanders RH. 1986. Astron. Astrophys. 158:297-304

Boyce PJ, Cohen RJ. 1994. Astron. Astrophys. Suppl. 107:563-647

Brown RL, Liszt HS. 1984. Annu. Rev. Astron. Astrophys. 22:223-65

Burton WB. 1992. In The Galactic Interstellar Medium, ed. D Pfenniger, P Bartholdi, pp. 
1-155. Berlin: Springer-Verlag. 400 pp.

Burton WB, Liszt HS. 1978. Ap. J. 228:815-42

Burton WB, Liszt HS. 1993. Astron. Astrophys. 274:765-74

Chudnovsky EM, Field GB, Spergel DN, Vilenkin A. 1986. Phys. Rev. D 34:944-50

Contopoulos G, Mertzanides C. 1977. Astron. Astrophys. 61:477-85

Conti PS. 1991. Ap. J. 377:115-25

Cook WR, Grunsfeld JM, Heindl WA, Palmer DM, Prince TA, et al. 1991. Ap. J. Lett. 372:L75-78

Cotera AS. 1995. Stellar ionization of the thermal emission regions in the Galactic center. $\mathrm{PhD}$ thesis. Stanford Univ. $165 \mathrm{pp}$.

Cotera AS, Erickson EF, Allen DA, Colgan SWJ, Simpson JP, Burton MG. 1994. See Genzel \& Harris 1994, pp. 217-21

Cotera AS, Erickson EF, Allen DA, Colgan SWJ, Simpson JP, Burton MG. 1995. In Airborne Astronomy Symp. on the Galactic Ecosystem: From Gas to Stars to Dust, ed. MR Haas, JA Davidson, EF Erickson, 73:511-14. San Francisco: ASP. 737 pp.

Cotera AS, Erickson EF, Colgan SWJ, Simpson JP, Allen DA, Burton MG. 1996. Ap. J. In press.

Cox P, Laureijs R. 1989. See Morris 1989, pp. $121-28$

Dame TM, Ungerechts H, Cohen RS, De Geus EJ, Grenier IA, et al. 1987. Ap. J. 322:706-20

Das M, Jog CJ. 1995. Ap. J. 451:167-75

Davidson JA. 1996. In Polarimetry of the Interstellar Medium, ed. WG Roberge, DCB Whittet. San Francisco: ASP. In press

Davidson JA, Werner MW, Wu X, Lester DF, Harvey PM, et al. 1992. Ap. J. 387:189-211

de Bruyn AG. 1976. Astron. Astrophys. 52:43947

de Vaucouleurs G. 1964. In IAU-URSI Symp. 20: The Galaxy and the Magellanic Clouds, ed. FJ Kerr, AW Rodgers, pp. 88-91, 195199. Canberra: Aust. Acad. Sci.

Dent WRF, Matthews HE, Wade R, Duncan WD. 1993. Ap. J. 410:650-62

Diehl R. 1994. See Genzel \& Harris 1994, pp. 3-12

Diehl R, Dupraz C, Bennett K, Bloemen H, Hermsen W, et al. 1995. Astron. Astrophys. 298:445-60

Duschl WJ. 1988. MNRAS 240:219-23

Duschl WJ, Lesch H. 1994. Astron. Astrophys. 286:431-6

Dwek E, Arendt RG, Hauser MG, Kelsall T, Lisse CM, et al. 1995. Ap. J. 445:716-30

Dyson JE, Hartquist TW. 1994. MNRAS 269:447-50

Echevarria L, Morris M. 1996. In preparation

Eckart A, Genzel R, Hofmann R, Sams BJ, Tacconi-Garman LE. 1993. Ap. J. Lett.
407:L77-80

Eckart A, Genzel R, Hofmann R, Sams BJ, Tacconi-Garman LE. 1995. Ap. J. Lett. 445:L23-26

Eckart A, Genzel R, Krabbe A, Hofmann R, van der Werf PP, Drapatz S. 1992. Nature 355:526-28

Ekers RD, van Gorkom JH, Schwarz UJ, Goss WM. 1983. Astron. Astrophys. 122:143-50

Falcke H. 1996. See Blitz \& Teuben 1996. p. 169

Falcke H, Heinrich OM. 1994. Astron. Astrophys. 292:430-38

Falcke H, Mannheim K, Biermann PL. 1993. Astron. Astrophys. 278:L1-4

Figer DF. 1995. A search for emission-line stars near the Galactic center. $\mathrm{PhD}$ thesis. Univ. Calif., Los Angeles. 214 pp.

Figer DF, McLean I, Morris M. 1995. Ap. J. Lett. 447:L29-32

Fleck RC. 1980. Ap. J. 242:1019-22

Frail DA, Diamond PJ, Cordes JM, Van Langevelde HJ. 1994. Ap. J. Lett. 427:L43-6

Fridman AM, Khoruzhii OV, Lyachovich VV, Ozernoy L, Blitz L. 1994. In Physics of the Gaseous and Stellar Disks of the Galaxy, ed. I King, pp. 285-303. San Francisco: ASP

Frogel JA. 1988. Annu. Rev. Astron. Astrophys. 26:51-92

Fukui Y, Churchwell E. 1987. See Backer 1987, pp. 110-13

Gatley I, Jones TJ, Hyland AR, Beattie DH, Lee TJ. 1984. MNRAS 210:565-75

Gatley I, Jones TJ, Hyland AR, Wade R, Geballe TR, Krisciunas TL. 1986. MNRAS 222:299306

Gatley I, Merrill M. 1994. In Infrared Astronomy with Arrays, ed. IS McLean, pp. 551-60. Dordrecht: Kluwer

Gaume RA, Claussen MJ, De Pree CG, Goss WM, Mehringer DM. 1995. Ap. J. 449:66373

Genzel R, Harris AI, eds. 1994. Nuclei of Normal Galaxies: Lessons from the Galactic Center. NATO ASI Ser. C. Dordrecht: Kluwer. 499 pp.

Genzel R, Hollenbach D, Townes CH. 1994. Rep. Prog. Phys. 57:417-79

Genzel R, Stacey GJ, Harris AI, Townes CH, Geis N, et al. 1990. Ap. J. 356:160-73

Genzel R, Townes CH. 1987. Annu. Rev. Astron. Astrophys. 25:377-423

Gezari D. 1992. In The Center, Bulge, and Disk of the Milky Way, ed. L Blitz, pp. 23-46. Dordrecht: Kluwer

Gezari D, Ozernoy L, Varosi F, McCreight C, Joyce R. 1994. See Genzel \& Harris 1994, pp. 427-30

Gerhard OE. 1992. Rev. Mod. Astron. 5:174-87

Gerhard OE. 1996. See Blitz \& Teuben 1996. p. 
79

Glass IS, Moneti A, Moorwood AFM. 1990. MNRAS 242:55-58p

Goldwurm A, Cordier B, Paul J, Ballet J, Bouchet L, et al. 1994. Nature 371:589-62

Goss WM, Schwarz UJ, van Gorkom JH, Ekers RD. 1985. MNRAS 215:69-73p

Gray AD, Cram LE, Ekers RD, Goss WM. 1991. Nature 353:237-39

Gray AD, Nicholls J, Ekers RD, Cram LE. 1995. Ap. J. 448:164-78

Güsten R. 1989. See Morris 1989, pp. 89-106

Güsten R, Downes D. 1980. Astron. Astrophys. 87:6-19

Güsten R, Downes D. 1981. Astron. Astrophys. 99:27-30

Güsten R, Genzel R, Wright MCH, Jaffe DT, Stutzki J, Harris AI. 1987. Ap. J. 318:124-38

Güsten R, Walmsley CM, Pauls T. 1981. Astron. Astrophys. 103:197-206

Gwinn CR, Danen RM, Middleditch J, Ozernoy LM, Tran TK. 1991. Ap. J. Lett. 381:L43-46

Haller JW, Rieke MJ, Rieke GH, Tamblyn P, Close L, Melia F. 1996. Ap. J. 456:194-205

Han C, Gould A. 1995. Ap. J. 449:521-26

Handa T, Sofue Y, Nakai N, Hirabayashi H, Inoue M. 1987. Publ. Astron. Soc. Jpn. 39:70953

Hasegawa T, Sato F, Whiteoak JB, Miyawaki R. 1994. Ap. J. Lett. 429:L77-80

Hauser MG, Silverberg RF, Stier MT, Kelsall T, Gezari DY, et al. 1984. Ap. J. 285:74-88

Haynes RF, Stewart RT, Gray AD, Reich W, Reich P, Mebold U. 1992. Astron. Astrophys. 264:500-12

Heiligman GM. 1987. Ap. J. 314:747-65

Herbst TM, Beckwith SVW, Forrest WJ, Pipher JL. 1993a. Astron. J. 105:956-70

Herbst TM, Beckwith SVW, Shure M. 1993 b. Ap. J. Lett. 411:L21-24

Heyvaerts J, Norman C, Pudritz RE. 1988. Ap. J. 330:718-36

Hildebrand R. 1988. Q. J. R. Astron. Soc. 29:327-51

Hildebrand RH, Davidson J, Dotson J, Figer DF, Novak G, et al. 1993. Ap. J. 417:565-71

Hildebrand RH, Gonatas DP, Platt SR, Wu XD, Davidson JA, et al. 1990. Ap. J. 362:114-19

Hills JG. 1975. Nature 254:295-98

Ho PTP. 1994. See Genzel \& Harris 1994, pp. 149-60

Ho PTP, Ho LC, Szczepanski JC, Jackson JM, Armstrong JT, Barrett AH. 1991. Nature 350:309-12

Hollywood JM, Melia F. 1995. Ap. J. Lett. 443:L17-20

Holt SS, McCray R. 1982. Annu. Rev. Astron. Astrophys. 20:323-65

Hüttemeister S, Wilson TL, Bania TM, MartinPintado J. 1993. Astron. Astrophys. 280:255-
67

Inoue M, Fomalont E, Tsuboi M, Yusef-Zadeh F, Morris M, et al. 1989. See Morris 1989, pp. 269-74

Izumiura H, Deguchi S, Hashimoto O, Nakada Y, Onaka T, et al. 1995. Ap. J. 453:837-63

Jackson JM, Geis N, Genzel R, Harris AI, Madden S, et al. 1993. Ap. J. 402:173-84

Jackson JM, Heyer MH, Paglione TAD, Bolatto AD. 1996. Ap. J. Lett. 456:L91-95

Jauncey DL, Tzioumis AK, Preston RA, Meier DL, Batchelor R. 1989. Astron. J. 98:44-48

Jenkins A, Binney J. 1994. MNRAS 270:703-19

Kaifu N, Kato T, Iguchi T. 1972. Nature Phys. Sci. 238:105-7

Kawai N, Fenimore EE, Middleditch J, Cruddace RG, Fritz GG, et al. 1988. Ap. J. 330:130-41

Kellermann KI, Pauliny-Toth IIK. 1981. Annu. Rev. Astron. Astrophys. 19:373-410

Kenney JDP, Wilson CD, Scoville NZ, Devereux NA, Young JS. 1992. Ap. J. Lett. 395:L79-82

Khokhlov A, Melia F. 1996. Ap. J. Lett. 457:L61-64

Killeen NEB, Lo KY. 1989. See Morris 1989, pp. $453-55$

Killeen NEB, Lo KY, Crutcher R. 1992. Ap. J. 385:585-603

Koyama K. 1996. See Blitz \& Teuben 1996. p. 287

Koyama K, Awaki H, Kunieda H, Takano S, Tawara Y, et al. 1989. Nature 339:603-05

Koyama K, Maeda Y, Sonobe T, Takeshima T, Tanaka Y, Yamauchi S. 1996. Publ. Astron. Soc. Jpn. In press

Krabbe A, Genzel R, Drapatz S, Rotaciuc V. 1991. Ap. J. Lett. 382:L19-22

Krabbe A, Genzel R, Eckart A, Najarro F, Lutz D, et al. 1995. Ap. J. Lett. 447:L95-99

Krichbaum TP, Schalinski CJ, Witzel A, Standke KJ, Graham DA, et al. 1994. See Genzel \& Harris 1994, pp. 411-14

Krichbaum TP, Zensus JA, Witzel A, Mezger PG, Standke KJ, et al. 1993. Astron. Astrophys. 274:L37-40

Lacey CG, Fall SM. 1985. Ap. J. 290:154-70

Lacy JH. 1994. See Genzel \& Harris 1994, pp. 165-74

Lacy JH, Achtermann JM, Bruce DE. 1989. See Morris 1989, pp. 523-24

Lacy JH, Achtermann JM, Serabyn E. 1991. Ap. J. Lett. 380:L71-74

Lacy JH, Townes CH, Geballe TR, Hollenbach DJ. 1980. Ap. J. 241:132-46

Lacy JH, Townes CH, Hollenbach DJ. 1982. Ap. J. 262:120-34

Lazarian A. 1994. MNRAS 268:713-23

Lee HM. 1994. See Genzel \& Harris 1994, pp. 335-42 
Lee HM. 1995. MNRAS 272:605-17

Lesch H, Crusius A, Schlickeiser R, Wielebinski R. 1989. Astron. Astrophys. 217:99-107

Lesch H, Reich W. 1992. Astron. Astrophys. 264:493-99

Lester DF, Bregman JD, Witteborn FC, Rank DM, Dinerstein HL. 1981. Ap. J. 248:52427

Lester DF, Dinerstein HL, Werner MW, Watson DM, Genzel R, Storey JWV. 1987. Ap. J. 320:573-85

Levine D. 1995. A survey of 22-GHz water masers within $2.25^{\circ}$ of the Galactic center. $\mathrm{PhD}$ thesis. Univ. Calif., Los Angeles. 290 pp.

Libonate S, Pipher JL, Forrest WJ, Ashby MLN. 1995. Ap. J. 439:202-23

Lindqvist M, Habing HJ, Winnberg A. 1992. Astron. Astrophys. 259:118-27

Lis DC, Carlstrom JE. 1994. Ap. J. 424:189-99

Lis DC, Menten KM, Serabyn E, Zylka R. 1994. Ap. J. Lett. 423:L39-42

Liszt HS. 1992. Ap. J. Suppl. 82:495-503

Liszt HS. 1996. See Blitz \& Teuben 1996. p. 297

Liszt HS, Burton WB. 1978. Ap. J. 226:790-816

Liszt HS, Burton WB. 1980. Ap. J. 236:779-97

Liszt HS, Burton WB. 1993. Ap. J. Lett. 407:L25-28

Liszt HS, Spiker RW. 1995. Ap. J. Suppl. 98:259-70

Liu T, Becklin EE, Henry JP, Simons D. 1993. Astron. J. 106:1484-89

Lo KY. 1989. See Morris 1989, pp. 527-34

Lo KY. 1994. See Genzel \& Harris 1994, pp. 395-402

Lo KY, Backer DC, Kellermann KI, Reid M, Zhao JH, et al. 1993. Nature 362:38-40

Lo KY, Claussen MJ. 1983. Nature 306:647-51

Lutz D, Krabbe A, Genzel R. 1993. Ap. J. 418:244-54

Lutz D, Krabbe A, Genzel R, Blietz M, Drapatz S, van der Werf PP. 1994. See Genzel \& Harris 1994, pp. 373-76

Maloney PR, Hollenbach DJ, Townes CH. 1992. Ap. J. 401:559-73

Marcaide JM, Alberdi A, Bartel N, Clark TA, Corey BE, et al. 1992. Astron. Astrophys. 258:295-301

Markevitch M, Sunyaev RA, Pavlinsky M. 1993. Nature 364:40-42

Marr JM, Rudolph AL, Pauls TA, Wright MC, Backer DC. 1992. Ap. J. Lett. 400:L29-32

Marr JM, Wright MC, Backer DC. 1993. Ap. J. 411:667-73

Marshall J, Lasenby AN. 1994a. See Genzel \& Harris 1994, pp. 175-77

Marshall J, Lasenby AN. 1994b. MNRAS 269:619-25

Marshall J, Lasenby AN, Yusef-Zadeh F. 1995.
MNRAS 274:519-22

Masset F, Tagger M. 1995. Astron. Astrophys. In press

Masset F, Tagger M. 1996. Astron. Astrophys. Submitted

Mastichiadis A, Ozernoy LM. 1994. Ap. J. 426:599-603

Mathis JS. 1990. Annu. Rev. Astron. Astrophys. 28:37-70

Mauersberger R, Henkel C, Wilson TL, Walmsley CM. 1986. Astron. Astrophys. 162:199210

McGinn MT, Sellgren K, Becklin EE, Hall DNB. 1989. Ap. J. 338:824-40

Meglicki Z, Wickramasinghe D, Dewar RL. 1994. MNRAS 272:717-29

Mehringer DM, Palmer P, Goss WM, YusefZadeh F. 1993. Ap. J. 412:684-95

Melia F. 1994a. Ap. J. 426:577-85

Melia F. 1994b. See Genzel \& Harris 1994, pp. 441-48

Mezger PG. 1978. Astron. Astrophys. 70:56574

Mezger PG, Pauls T. 1979. In IAU Symp. No. 84, The Large-scale Characteristics of the Galaxy, ed. WB Burton, pp. 357-66. Dordrecht: Reidel

Mezger PG, Zylka R, Salter CJ, Wink JE, Chini R, et al. 1989. Astron. Astrophys. 209:33748

Miller RH, Smith BF. 1992. Ap. J. 393:508-15

Moneti A, Glass I, Moorwood A. 1992. MNRAS 258:705-14

Moneti A, Glass I, Moorwood A. 1994. MNRAS 268:194-202

Morris M, ed. 1989. IAU Symp. No. 136, The Center of the Galaxy. Dordrecht: Kluwer. $661 \mathrm{pp}$.

Morris M. 1990. In IAU Symp. No. 140, Galactic and Extragalactic Magnetic Fields, ed. R Beck, P Kronberg, R Wielebinski, pp. 36167. Dordrecht: Kluwer

Morris M. 1993. Ap. J. 408:496-506

Morris M. 1994. See Genzel \& Harris 1994, pp. 185-98

Morris M. 1996. See Blitz \& Teuben 1996. p. 247

Morris M, Davidson JA, Werner MW. 1995. In Airborne Astronomy Symposium on the Galactic Ecosystem, ed. MR Haas, JA Davidson, EF Erickson, ASP Conf. Ser. 73:477-88. San Francisco: ASP

Morris M, Davidson JA, Werner MW, Dotson J, Figer DF, et al. 1992. Ap. J. Lett. 399:L6366

Morris M, Davidson JA, Werner MW, Hildebrand RH, Dotson J, et al. 1996a,b. In preparation

Morris M, Polish N, Zuckerman B, Kaifu N. 1983. Astron. J. 88:1228-35 
Morris M, Yusef-Zadeh F. 1985. Astron. J. 90:2511-13

Morris M, Yusef-Zadeh F. 1987. See Backer 1987, pp. 127-32

Morris M, Yusef-Zadeh F. 1989. Ap. J. 343:70312

Mulder WA, Liem BT. 1986. Astron. Astrophys. 157:148-58

Nagata T, Woodward CE, Shure M, Kobayashi N. 1995. Astron. J. 109:1676-81

Nagata T, Woodward CE, Shure M, Pipher JL, Okuda H. 1990. Ap. J. 351:83-88

Nakada Y, Deguchi S, Hashimoto O, Izumiura H, Onaka T, et al. 1991. Nature 353:140-41

Nakagawa T, Doi Y, Yui YY, Okuda H, Mochizuki K, et al. 1996. Ap. J. Lett. In press

Narayan R, Yi I. 1995. Ap. J. 452:710-35

Narayan R, Yi I, Mahadevan R. 1995. Nature 374:623-25

Nottingham MR, Skinner GK, Willmore AP, Borozdin KN, Churazov E, Sunyaev RA. 1993. Astron. Astrophys. Suppl. 97:165-67

Odenwald SF, Fazio GG. 1984. Ap. J. 283:60114

Oka T, Hasegawa T, Handa T, Hayashi M, Sakamoto S. 1996. Ap. J. 460:334-342

Okuda H, Shibai H, Nakagawa T, Matsuhara H, Kobayashi Y, Kaifu N, Nagata T, Gatley I, Geballe TR. 1990. Ap. J. 351:89-97

Oort J. 1977. Аnnu. Rev. Astron. Astrophys., 15:295-362

Owen FN, Spangler SR, Cotton WD. 1980. Astron. J. 85:351-62

Ozernoi LM. 1979. In Large-Scale Characteristics of the Galaxy, ed. WB Burton, pp. 395400. Dordrecht: Reidel

Ozernoy LM. 1989. See Morris 1989, pp. 55566

Ozernoy LM. 1993. In Back to the Galaxy, ed. SS Holt, F Verter, pp. 69-72. New York: AIP

Ozernoy LM. 1994. See Genzel \& Harris 1994, pp. 431-40

Paczyński B, Stanek KZ, Udalski A, Szymański M, Kaluzny J, et al. 1994. Ap. J. Lett. 435:L113-16

Pak S, Jaffe DT, Keller LD. 1996. Ap. J. Lett. 457:L43-46

Pauls T, Johnston KJ, Wilson TL, Marr JM, Rudolph A. 1993. Ap. J. Lett. 403:L13-16

Pavlinsky MN, Grebenev SA, Sunyaev RA. 1994. Ap. J. 425:110-21

Pedlar A, Anantharamaiah KR, Ekers RD, Goss WM, van Gorkom JH, et al. 1989. Ap. J. 342:769-84

Peters WL. 1975. Ap. J. 195:617-29

Phinney ES. 1989. See Morris 1989, pp. 543-53

Pietsch W. 1994. See Genzel \& Harris 1994, pp 13-19

Plante RL, Lo KY, Crutcher RM. 1995. Ap. J. Lett. 445:L113-16
Poglitsch A, Stacey GJ, Geis N, Haggerty M, Jackson J, et al. 1991. Ap. J. Lett. 374:L3336

Predehl P, Trümper J. 1994. Astron. Astrophys. 290:L29-32

Quinn PJ, Sussman GJ. 1985. Ap. J. 288:377-84

Rees MJ. 1988. Nature 333:523-28

Rees MJ, Begelman MC, Blandford RD, Phinney ES. 1982. Nature 295:17-21

Reich W. 1994. See Genzel \& Harris 1994, pp 55-62

Reich W, Sofue Y, Fürst E. 1987. Publ. Astron. Soc. Jpn. 39:573-87

Reid M. 1993. Annu. Rev. Astron. Astrophys. 31:345-72

Reynolds SP, McKee CF. 1980. Ap. J. 239:89397

Rich RM. 1993. In IAU Symp. No. 153. Galactic Bulges, ed. HJ Habing, T DeJonghe, pp. 19. Dordrecht: Kluwer

Riegler GR, Blandford RD, eds. 1982. AIP Conf. Proc. No. 83, The Galactic Center. New York: AIP. 216 pp.

Rieke GH, Lebofsky MJ. 1982. See Riegler \& Blandford 1982, pp. 194-203

Rieke GH, Lebofsky MJ. 1985. Ap. J. 288:61821

Rieke GH, Lebofsky MJ. 1987. See Backer 1987, pp. 91-94

Rieke GH, Rieke MJ. 1988. Ap. J. Lett. 330:L33-37

Rieke GH, Rieke MJ. 1989. Ap. J. Lett. 344:L58

Rieke GH, Rieke MJ. 1994. See Genzel \& Harris 1994, pp. 283-91

Rieke GH, Rieke MJ, Paul AE. 1989. Ap. J. 336:752-61

Rieke MJ. 1993. In Back to the Galaxy, ed. SS Holt, F Verter, pp. 37-42. New York: AIP

Roberge WG, Hanany S, Messinger DW. 1995. Ap. J. 453:238-55

Roberts DA, Goss WM. 1993. Ap. J. Suppl. 86:113-52

Rogers AEE, Doeleman S, Wright $\mathrm{MCH}$, Bower GC, Backer DC, et al. 1994. Ap. J. Lett. 434:L59-62

Rosso F, Pelletier G. 1993. Astron. Astrophys. 270:416-25

Saito M. 1990. Publ. Astron. Soc. Jpn. 42:19-38

Sanders RH. 1981. Nature 294:427-29

Sanders RH. 1989. See Morris 1989, pp. 77-87

Schwarz UJ, Lasenby J. 1990. In IAU Symp. No. 170, Galactic and Intergalactic Magnetic Fields, ed. R Beck, PP Kronberg, R Wielebinski, pp. 383-84, Dordrecht: Kluwer

Scoville NZ. 1972. Ap. J. Lett. 175:L127-32

Scoville NZ, Solomon PM, Jefferts KB. 1974. Ap. J. Lett. 187:L63-66

Scoville NZ, Good JC. 1989. Ap. J. 339:149-62

Scoville NZ, Sanders DB. 1987. In Interstellar 
Processes, ed. DJ Hollenbach, HA Thronson, pp. 21-50. Dordrecht: Reidel

Sellgren K, Hall DN, Kleinmann SG, Scoville NZ. 1987. Ap. J. 317:881-91

Sellgren K, McGinn MT, Becklin EE, Hall DNB. 1990. Ap. J. 359:112-20

Sellwood JA. 1993. In Back to the Galaxy, ed. SS Holt, F Verter, pp. 133-36. New York: AIP

Serabyn E. 1984. PhD dissertation. Univ. Calif., Berkeley

Serabyn E. 1989. See Morris 1989, pp. 437-41

Serabyn E. 1996. See Blitz \& Teuben 1996. p. 263

Serabyn E, Carlstrom JE, Scoville NZ. 1992a. Ap. J. Lett. 401:L87-88

Serabyn E, Carlstrom JE, Scoville NZ, Lis DC, Lacy JH. 1996a. In prep.

Serabyn E, Güsten R. 1987. Astron. Astrophys 184:133-43

Serabyn E, Güsten R. 1991. Astron. Astrophys 242:376-87

Serabyn E, Güsten R, Walmsley CM, Wink JE, Zylka R. 1986. Astron. Astrophys 169:85-94

Serabyn E, Keene J, Lis DC, Phillips TG. 1994 Ap. J. Lett. 424:L95-98

Serabyn E, Keene J, Lis DC, Phillips TG. 1996 b. In prep.

Serabyn E, Lacy JH. 1985. Ap. J. 293:445-58

Serabyn E, Lacy JH, Achtermann JM. 1991. Ap. J. 378:557-64

Serabyn E, Lacy JH, Achtermann JM. 1992 b. Ap. J. 395:166-73

Serabyn E, Lacy JH, Townes CH, Bharat R. 1988. Ap. J. 326:171-85

Serabyn E, Morris M. 1994. Ap. J. Lett. 424:L91-94

Serabyn E, Shupe D. 1996. In prep.

Shibata K, Uchida Y. 1987. Publ. Astron. Soc. Jpn. 39:559-71

Shields JC, Ferland GJ. 1984. Ap. J. 430:236-51

Shlosman I, Frank J, Begelman MC. 1989. Nature 338:45-47

Sjouwerman LO, van Langevelde HJ. 1996. Ap. J. Lett. 461:L41-44

Skinner GK. 1989. See Morris 1989, pp. 56780

Smith CH, Aitken DK, Roche PF. 1990. MNRAS 246:1-9

Sofue Y. 1994. See Genzel \& Harris 1994, pp. 43-54

Sofue Y. 1995a. Publ. Astron. Soc. Jpn. 47:52749

Sofue Y. 1995b. Publ. Astron. Soc. Jpn. 47:55159

Sofue Y, Fujimoto M. 1987. Publ. Astron. Soc. Jpn. 39:843-48

Sofue Y, Murata Y, Reich W. 1992. Publ. Astron. Soc. Jpn. 44:367-72

Sofue Y, Reich W, Inoue M, Seiradakis JH.
1987. Publ. Astron. Soc. Jpn. 39:95-107

Sofue Y, Reich W, Reich P. 1989. Ap. J. Lett. 341:L47-49

Sparke LS. 1984. MNRAS 211:911-26

Spergel DN, Blitz L. 1992. Nature 357:665-67

Staguhn J, Stutzki J, Yusef-Zadeh F, Uchida KI. 1996. In preparation

Stanek KZ, Mateo M, Udalski A, Szymański J, Kaluzny J, Kubiak M. 1994. Ap. J. Lett. 429:L73-6

Stark AA, Bally J, Wilson RW, Pound MW. 1989. See Morris 1989, pp. 129-33

Stark AA, Bania TM. 1986. Ap. J. Lett. 306:L17-20

Stark AA, Gerhard OE, Binney J, Bally J. 1991. MNRAS 248:14-17p

Stolovy S, Hayward T, Herter T. 1996. In prep.

Sunyaev R, Markevitch M, Pavlinsky M. 1993. Ap. J. 407:606-10

Sutton EC, Danchi WC, Jaminet PA, Masson CR. 1990. Ap. J. 348:503-14

Syunyaev R, Pavlinskii M, Gil'fanov M, Churazzov E, Grebenev S, et al. 1991. Sov. Astron. Lett. 17:42-45

Tagger, M, Athanassoula, E. 1990. In Dynamics of Galaxies and Their Molecular Cloud Distributions, ed. F Combes, F Casoli, pp. 105-07. Dordrecht: Kluwer

Tamblyn P, Rieke GH. 1993. Ap. J. 414:573-79

Tamblyn P, Rieke GH, Hanson MM, Close LM, McCarthy DW Jr, Rieke MJ. 1996. Ap. J. 456:206-16

Taylor GB, Morris M, Schulman E. 1993. Astron. J. 106:1978-86

Telesco CM, Davidson JA, Werner MW. 1996. Ap. J. 456:541-56

Terlevich R, Melnick J. 1985. MNRAS 213:84156

Tsuboi M, Handa T, Inoue M, Inatani J, Ukita N. 1989. See Morris 1989, pp. 135-40

Tsuboi M, Inoue M, Handa T, Tabara H, Kato T, et al. 1986. Astron. J. 92:818-24

Tsuboi M, Kawabata T, Kasuga T, Handa T, Kato T. 1995. Publ. Astron. Soc. Jpn. 47:82936

Turner JL, Hurt RL, Hudson DY. 1993. Ap. J. Lett. 413:L19-22

Uchida KI. 1993. The manifestations of energetic activity in the Galactic center. $\mathrm{PhD}$ thesis. Univ. Calif., Los Angeles. 258 pp.

Uchida KI, Güsten R. 1995. Astron. Astrophys. 298:473-81

Uchida KI, Morris M, Bally J. 1994a. See Genzel \& Harris 1994, pp. 99-103

Uchida KI, Morris M, Bally J. 1996a. In preparation

Uchida KI, Morris M, Güsten R, Serabyn E. 1996b. Ap. J. 462:768-76

Uchida KI, Morris M, Serabyn E, Bally J. 1994b. Ap. J. 421:505-16 
Uchida Y, Shibata K, Sofue Y. 1985. Nature 317:699-701

Umemura S, Iki K, Shibata K, Sofue Y. 1988. Publ. Astron. Soc. Jpn. 40:25-46

von Linden S, Duschl WJ, Biermann PL. 1993. Astron. Astrophys. 269:169-74

Wada K, Taniguchi Y, Habe A, Hasegawa T. 1994. Ap. J. Lett. 437:L123-25

Walmsley CM, Güsten R, Angerhofer P, Churchwell E, Mundy L. 1986. Astron. Astrophys. 155:129-36

Wannier PG. 1989. See Morris 1989, pp. 10719

Wardle M, Königl A. 1990. Ap. J. 362:120-34

Wardle M, Yusef-Zadeh F. 1992. Nature 357:308-10

Watson MG, Willingale R, Grindlay JE, Hertz P. 1981. Ap. J. 250:142-54

Weiland JL. et al. 1994. Ap. J. Lett. 425:L81-84

Weinberg MD. 1992a. Ap. J. 384:81-94

Weinberg MD. 1992b. Ap. J. Lett. 392:L67-69

Werner MW, Davidson JA, Morris M, Novak G, Platt SR, Hildebrand RH. 1988. Ap. J. 333:729-34

Whitelock P, Catchpole R. 1992. In The Center, Bulge, and Disk of the Milky Way, ed. L Blitz, pp. 103-10. Dordrecht: Kluwer

Wilson TL, Ruf K, Walmsley CM, Martin RN, Pauls TA, Batrla W. 1982. Astron. Astrophys. 115:185-89

Wright EL, Mather JC, Bennet CL, Cheng ES, Shafer RA, et al. 1991. Ap. J. 381:200-9

Wright MCH, Genzel R, Güsten R, Jaffe, DT. 1987. See Backer 1987, pp. 133-37

Wright $\mathrm{MCH}$, Backer DC. 1993. Ap. J. 417:560-64

Yamauchi S, Kawada M, Koyama K, Kunieda H, Tawara Y, Hatsukade I. 1990. Ap. J. 365:532-38

Yamauchi S, Koyama K. 1993. Ap. J. 404:62024

Yusef-Zadeh F. 1989. See Morris 1989, pp. 243 63

Yusef-Zadeh F. 1994. See Genzel \& Harris 1994, pp. 355-72

Yusef-Zadeh F, Cotton W, Wardle M, Melia F,
Roberts DA. 1994. Ap. J. 434:L63-6

Yusef-Zadeh F, Lasenby A, Marshall J. 1993. Ap. J. Lett. 410:L27-30

Yusef-Zadeh F, Mehringer DM. 1995. Ap. J. Lett. 452:L37-40

Yusef-Zadeh F, Melia F. 1992. Ap. J. Lett. 385:L41-44

Yusef-Zadeh F, Morris M. 1987a. Ap. J. 320:545-61

Yusef-Zadeh F, Morris M. 1987b. Astron. J. 94:1178-84

Yusef-Zadeh F, Morris M. 1987c. Ap. J. 322:721-28

Yusef-Zadeh F, Morris M. 1988. Ap. J. 329:729_ 38

Yusef-Zadeh F, Morris M. 1991. Ap. J. Lett. 371:L59-62

Yusef-Zadeh F, Morris M, Chance D 1984. Nature 310:557-61

Yusef-Zadeh F, Morris M, Ekers RD. 1989. See Morris 1989, pp. 443-51

Yusef-Zadeh F, Morris M, Ekers RD. 1990. Nature 348:45-47

Yusef-Zadeh F, Wardle M. 1993. Ap. J. 405:584-90

Yusef-Zadeh F, Zhao JH, Goss WM. 1995. Ap. J. 442:646-52

Zhao J-H, Ekers RD, Goss WM, Lo KY. 1989. See Morris, pp. 535-41

Zhao J-H, Goss WM, Ho PTP. 1995. Ap. J. 450:122-36

Zhao J-H, Goss WM, Lo KY, Ekers RD. 1992. In Relationships between Active Galatic Nuclei and Starburst Galaxies, ed. AV Filipenko, pp. 295-99. San Francisco: ASP

Zhao H-S, Spergel DN, Rich RM. 1994. Astron. J. 108:2154-63

Zylka R, Mezger PG. 1988. Astron. Astrophys. 190:L25-28

Zylka R, Mezger PG, Lesch H. 1992. Astron. Astrophys. 261:119-29

Zylka R, Mezger PG, Ward-Thompson D, Duschl WJ, Lesch H. 1995. Astron. Astrophys. 297:83-97

Zylka R, Mezger PG, Wink JE. 1990. Astron. Astrophys. 234:133-46 


\footnotetext{
A Annual Review of Astronomy and Astrophysics

R Volume 34 (1996)

;
}

\section{CONTENTS}

THE DESIRE TO UNDERSTAND THE WORLD, Evry Schatzman

Corotating and TRansient Solar Wind Flows In THREE

DIMENSIONS, J. T. Gosling

Solar ACtive Regions as Diagnostics of Subsurface

CONDITIONS, Robert F. Howard

BIPOLAR MOLECULAR OUTFLOWS FROM YOUNG STARS AND PROTOSTARS, Rafael Bachiller

Galactic MaGnetism: ReCENT DEVELOPMENTS AND PERSPECTIVES, Rainer Beck, Axel Brandenburg, David Moss, Anvar Shukurov, Dmitry Sokoloff

The FU ORIonis Phenomenon, Lee Hartmann, Scott J. Kenyon 207

CIRCUMSTELlar Photochemistry, A. E. Glassgold

INTERSTELLAR ABUNDANCES FROM ABSORPTION-LINE OBSERVATIONS WITH THE HUBbLE SPACE TELESCOPE, Blair D. Savage, Kenneth R. Sembach

Geminga: its Phenomenology, ITS Fraternity, AND ITs Physics, Giovanni F. Bignami, Patrizia A. Caraveo

Charged Dust DyNAmics IN THE Solar System, Mihaly Horányi

GRAVITATIONAL MiCROLENSING IN THE LOCAL GROUP, Bohdan Paczyński

The Age of the Galactic Globular Cluster System, Don A. VandenBerg, Peter B. Stetson, Michael Bolte

Old AND INTERMEdiate-Age STELlaR Populations IN THE MAGELlaniC Clouds, Edward W. Olszewski, Nicholas $B$. Suntzeff, Mario Mateo

Stellar PUlSATIONS ACROSS THE HR Diagram: Part 2, Alfred Gautschy, Hideyuki Saio

X-RAY NovaE, Y. Tanaka, N. Shibazaki

THE GalaCTIC CENTER ENVIRONMENT, Mark Morris, Gene Serabyn 
THEORY OF ACCRETION DISKS II: APPLICATION TO OBSERVED Systems, D. N. C. Lin, J. C. B. Papaloizou 703

Luminous InFRAREd Galaxies, D. B. Sanders, I. F. Mirabel 749 INDEXES

Subject Index

Cumulative Index of Contributing Authors, Volumes 24-34

\section{SOME RELATED ARTICLES IN OTHER ANNUAL REVIEWS}

From the Annual Review of Earth and Planetary Sciences, Volume 23 (1995):

Radar Observations of Mars, Mercury, and Titan, DO Muhleman, AW Grossman, and BJ Butler

The Origin of Life in the Solar System: Current Issues, CF Chyba and GD McDonald

Volume 24 (1996)

Io on the Eve of the Galileo Mission, JR Spencer and NM Schneider

Probing Planetary Atmospheres with Stellar Occulations, JL Elliot and CB Olkin

From the Annual Review of Nuclear and Particle Science,

Volume 44 (1994)

Astrophysical Sources of Gravitational Radiation, S Bonazzola and J-A Marck

Volume 45 (1995)

Matter at Large Neutron Excess and the Physics of Neutron Star Crusts, CJ Pethick and G Ravenhill 